\title{
Pleistozän-Geologie und Palynostratigraphie in Subrosionssenken Ostwestfalens
}

\author{
OtFried Deutloff \& RÜdiger STRITZKE*) \\ - Lower to Upper Pleistocene, subrosion depressions, typical clastic sediments, \\ pollenanalytical dating, stratigraphy, Eastern Westphalia -
}

\begin{abstract}
Kurzfassung: Im ostwestfälischen Bergland entstanden seit der Tertiärzeit durch Auslaugung von Salinargesteinen (Steinsalz und Anhydrit) im Untergrund zahlreiche Subrosionssenken an der Erdoberfläche. In diesen Senken sammelten sich während des Tertiärs und Quartärs verschiedenartige Lockergesteine - Sande, Silte, Tone -, die hier vor Erosion geschützt waren.

Dank systematischer Erkundung während der Neukartierung Ostwestfalens durch das Geologische Landesamt Nordrhein-Westfalen wurden bisher rund 80 Subrosionssenken zumeist durch Bohrungen entdeckt. Zehn von ihnen werden in der vorliegenden Arbeit beschrieben, wobei acht nach palynologischer Datierung warmzeitliche Sedimente des Unterpleistozän, Cromer, Holstein und Eem beinhalten, weitere zwei solche der Kaltzeiten Saale und womöglich Elster. Die mächtigste und vollständigste Abfolge vom Waal bis zum Cromer wurde in der Subrosionssenke von Mosebeck östlich Detmold angetroffen.
\end{abstract}

\section{[Pleistocene geology and palynostratigraphy in subrosion depressions of East Westphalia]}

\begin{abstract}
In the East Westphalian mountain ridges numerous subrosion depressions were built due to partial leaching of saliniferous rocks (halite and anhydrite) since Tertiary times. They acted as sedimentary traps and accumulated mainly clastic rocks since then. In the course of modern mapping in Eastern Westphalia members of the Geological Survey of Northrhine Westphalia discovered approximately 80 subrosion depressions mainly by drilling. Ten of those are here described, where 8 contained interglacial sediments of Lower Pleistocene, Cromerian, Holsteinian and Eemian age due to palynological investigations, whereas in two of them glacial sediments of the Saalian and probably of the Elsterian were preserved. The most complete (Lower to Middle Pleistocene) and thickest sequence of Pleistocene sediments was found in the subrosion depression of Mosebeck near Detmold.
\end{abstract}

\section{Einleitung}

Das Hauptverbreitungsgebiet pleistozäner Schichten im nördlichen Deutschland ist der Teil-

*) Anschriften der Verfasser: Dr. O. Deutloff, Ortmannsweg 3, 47918 Tönisvorst, Dr. R. STRITZKE, Geologisches Landesamt NRW, PF 1080, D-47710 Krefeld bereich des Mitteleuropäischen Tieflands zwischen Oder und Niederrhein mit seinen zum Teil mächtigen kalt- und warmzeitlichen Schichtenfolgen. Die kaltzeitlichen Ablagerungen gehen dabei auf den mehrmaligen Vorstoß des skandinavischen Inlandeises zurück, das mit seinen Gletschern entlang den Flußtälern von Elbe und Saale bis weit nach Süden in die Mittelgebirgsregionen zwischen Lausitz und Thüringer Becken vordrang (Elster- und Saale-Kaltzeit). Dort wurden durch die ausgedehnten Braunkohlentagebaue mächtige, stark gegliederte pleistozäne Sedimentfolgen aufgeschlossen (EIssmann 1994).

Auch im Bereich der nordwestdeutschen Tiefebene zwischen Elbe und Ems sowie in den Niederlanden sind entsprechende Ablagerungen anzutreffen. Sie sind dort insbesondere aus der Untersuchung tief reichender Bohrungen bekannt. Am südlich anschließenden Gebirgsrand und in den Mittelgebirgen selbst wurden Sedimente des Pleistozäns vorwiegend oder ausschließlich in den als Sedimentfallen wirksamen Subrosionssenken (= Auslaugungssenken) über leicht löslichen Salinargesteinen im Untergrund abgelagert und erhalten. Hierbei handelt es sich in der Regel um mächtige fein- bis grobklastische, teilweise humose Schichtenfolgen, welche die vielgestaltigen geologischen Prozesse während des Quartärs widerspiegeln. Derartige Sedimentfolgen wurden erstmalig durch H. WEBER (1952) aus dem Pliozän des Werragebietes in Westthüringen beschrieben, später aus dem Südniedersächsischen Bergland (z. B. BENDA et al. 1968, LÜTTIG 1969) und als unerwartete Ergebnisse von Brunnenbohrungen auch aus dem ostwestfälischen Raum mitgeteilt (Mestwerdt 1951, BECKER 1975, SCHNEIDER 1975).

Nachdem die Bedeutung der Subrosionssenken für die geologische Praxis erkannt worden war, wurden bei der Neukartierung des nordrheinwestfälischen Weserberglandes durch das Geologische Landesamt Nordrhein-Westfalen dank systematischer Erkundung bisher insgesamt rund 
80 Senken mit teilweise tertiärzeitlicher, überwiegend jedoch quartärzeitlicher Sedimentfüllung bekannt. Eine erste Übersicht über die bis dahin entdeckten Subrosionssenken findet sich im Tätigkeitsbericht 1984 bis 1985 des Geologischen Landesamtes Nordrhein-Westfalen (Abb. 24). Detaillierter dargestellt und beschrieben werden sie in der Geologischen Karte von NordrheinWestfalen im Maßstab 1:100000, Blätter C 3918 Minden (Deutloff et al. 1982) und C 3914 Bielefeld (Deutloff, KühN-Velten \& Michel 1986) sowie in den neu bearbeiteten Geologischen Karten von Nordrhein-Westfalen im Maßstab 1:25000, Blätter 3918 Bad Salzuflen (KNaufF 1978), 4019
Detmold (FARRENSCHON 1986), 3818 Herford (DeutlofF 1995b), 4020 Blomberg (FARRENSCHON 1995b) und 3919 Lemgo (FARRENSCHON 1998). In etwa 20 Subrosionssenken konnten die dort angetroffenen humosen Lockergesteine mittels Pollenanalyse stratigraphisch eingestuft werden. Die Hauptschwierigkeit bei der palynologischen Einstufung lag darin, daß aus den Subrosionssenken nicht ausschließlich Seesedimente zur Verfügung standen, sondern Material verschiedener Genese.

Eine Datierung ist hierdurch naturgemäß schwierig, zumal auch aus der näheren Umgebung sichere Standardprofile fehlen.
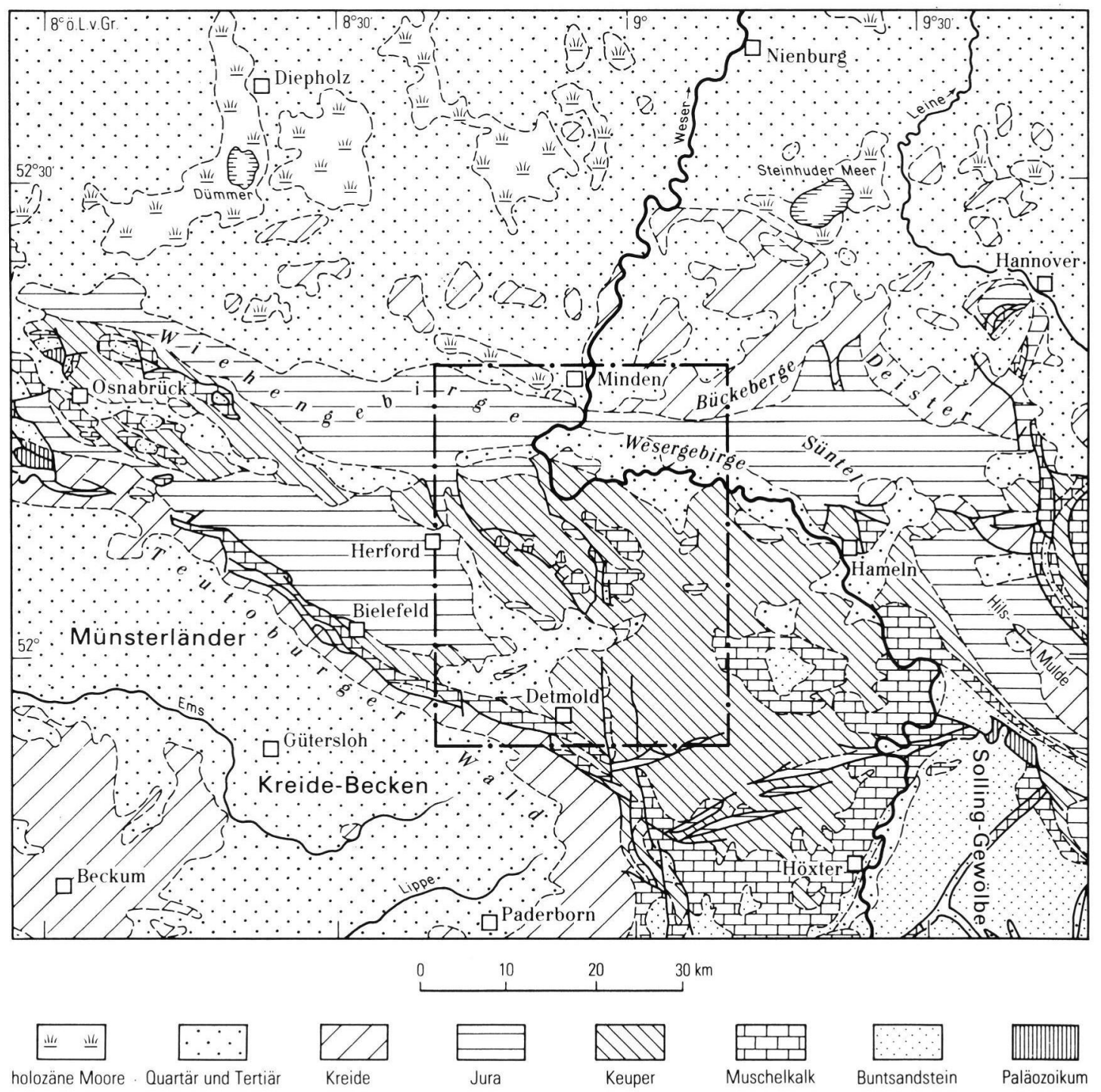

Abb. 1: Lage des Arbeitsgebietes in Ostwestfalen.

Fig. 1: Location of the investigation area in Eastern Westphalia. 


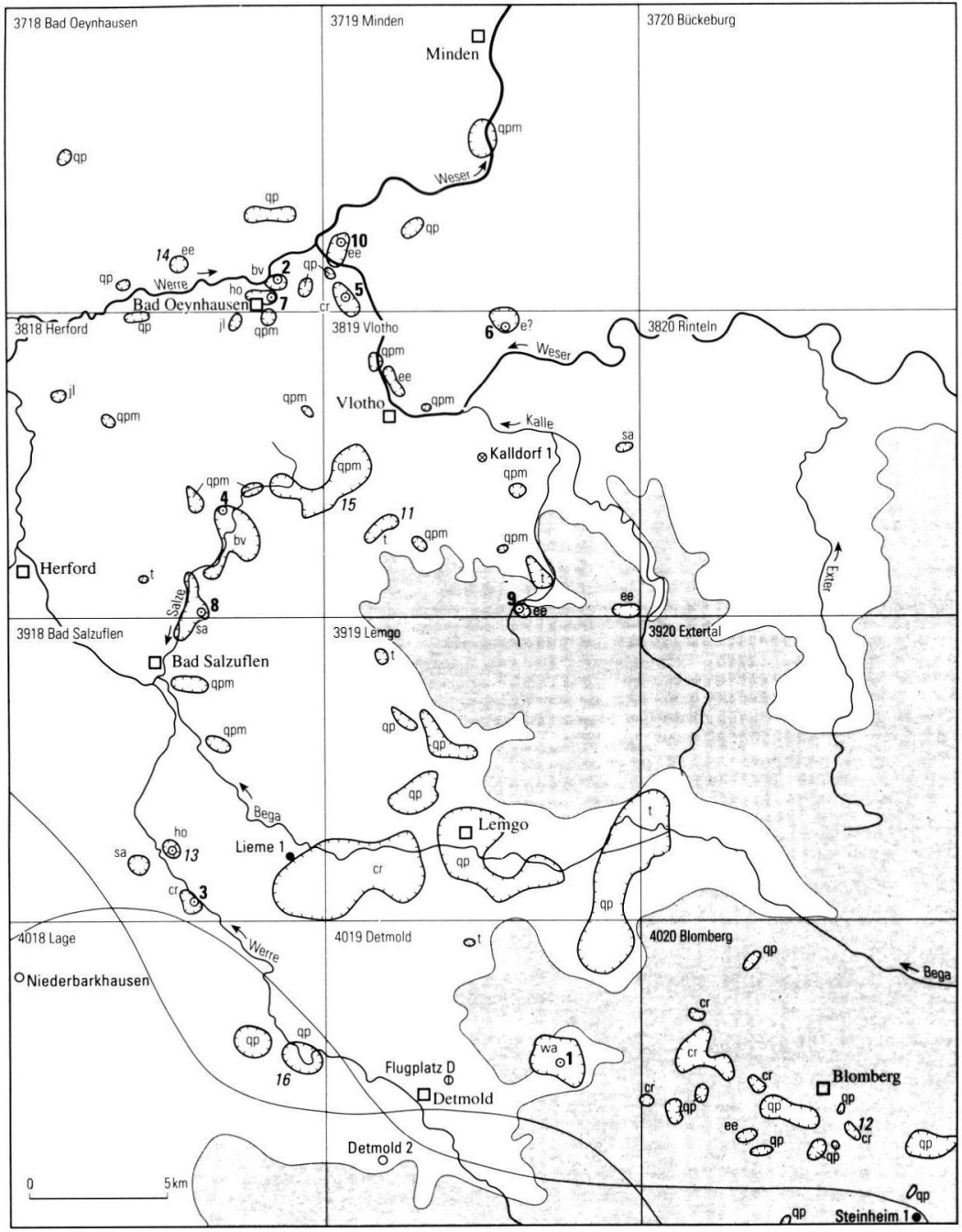

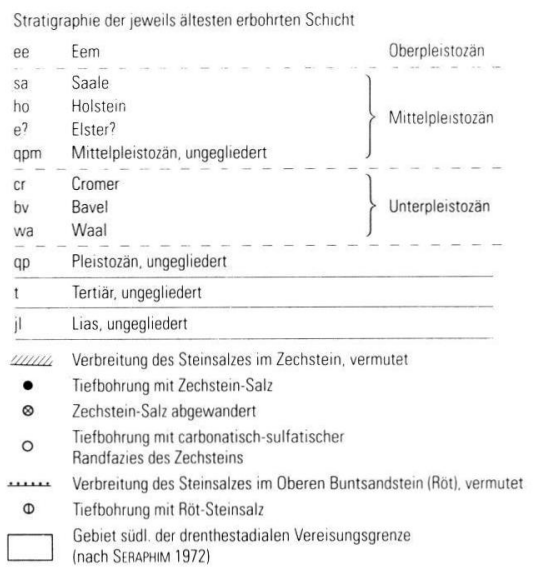

Den Bohrungen wurden die Proben zur Pollenanalyse unter Berücksichtigung der Petrographie möglichst engständig entnommen, im Labor mit Kalilauge und 45\%iger Flußsäure sowie nach der AcetolyseMethode von Erdtmann aufbereitet und abschließend gesiebt (10 $\mu \mathrm{m})$. Die Pollendichte war oft gering, so daß je Probe meist mehrere Präparate gezählt werden mußten, um statistisch ausreichende Pollenmengen zu ermitteln. Dabei geschah die Bestimmung in Anlehnung an die Bestimmungsschlüssel von MOORE et al. (1991) sowie FAEGRI et al. (1993). Die Ergebnisse der Pollenanalyse sind in Pollendiagrammen dargestellt, wobei das Programm psimpoll von BENNET (1994) verwandt wurde.

Dabei beziehen sich die prozentualen Angaben auf die Summe aller Landpflanzenpollen = $100 \%$. Die Abszisse hat für alle Pollen- und Sporentypen stets den gleichen Maßstab: ein Skalenstrich entspricht $20 \%$. Bei Werten unter 5\% wurde ein Punkt gesetzt.

Abb. 2: Lage und Alter der bisher bekannten Subrosionssenken im Arbeitsgebiet.

Fig. 2: Location and age of the hitherto known subrosion depressions in the investigation area. 
Tabelle 1: Die wichtigsten Daten der beschriebenen Subrosionssenken.

Table 1: The most important facts of the subrosion depressions described.

\begin{tabular}{|c|c|c|c|c|c|c|c|c|c|c|c|}
\hline 这 & Name & TY ? & $\sum_{\substack{z \\
0}}$ & $\stackrel{ \pm}{\sim}$ & & wichtiges & Festgestein & R & La & ge & 용 \\
\hline 믁 & Subrosionssenke & & 욷 트 & 茜㙜 & $\cong \bar{E}$ & altestes Quartär & Liegenden & & $R$ & H & \\
\hline 1 & Mosebeck & $\begin{array}{c}4019 \\
\text { Detmold }\end{array}$ & 160 & 3,00 & 139 & $\begin{array}{c}\text { Cromer } \\
\text { bis } \\
\text { Waal B }\end{array}$ & Oberer Keuper & $\begin{array}{c}\text { KB Mosebeck } \\
1976\end{array}$ & 96880 & 57120 & 141,8 \\
\hline 2 & $\begin{array}{c}\text { Schlachthof } \\
\text { Bad Oeynhausen }\end{array}$ & $\begin{array}{c}3718 \\
\text { Bad Oeynhausen }\end{array}$ & 52 & 0.09 & 28 & Bavel & $\begin{array}{l}\text { Unterer Lias } \\
\text { (Sinemur) }\end{array}$ & $\begin{array}{c}\text { KB } 10 / \text { A } 30 \\
1985\end{array}$ & 87019 & 86269 & 50,0 \\
\hline 3 & $\begin{array}{l}\text { Iggenhausener } \\
\text { Wald }\end{array}$ & $\begin{array}{c}3918 \\
\text { Bad Salzuflen }\end{array}$ & 95 & 0.36 & 60 & $\begin{array}{l}\text { Saale } \\
\text { und } \\
\text { Holstein }\end{array}$ & $\begin{array}{l}\text { Unterer Lias } \\
\text { (Hettang) }\end{array}$ & $\begin{array}{c}\text { KB Iggenhausen } \\
1989\end{array}$ & 83800 & 63560 & 61,0 \\
\hline 4 & Exter-Krutheide & $\begin{array}{l}3818 \\
\text { Herford }\end{array}$ & 110 & 2,50 & 53 & $\begin{array}{l}\text { Cromer } \\
\text { und } \\
\text { Bavel }\end{array}$ & Schilfsandstein & $\begin{array}{l}\text { KB Exter } \\
1989\end{array}$ & 84920 & 77800 & 54,0 \\
\hline 5 & Babbenhausen & $\begin{array}{c}3719 \\
\text { Minden u. }\end{array}$ Vlotho & 85 & 0,30 & 50 & $\begin{array}{l}\text { Saale } \\
\text { und } \\
\text { Cromer }\end{array}$ & $\begin{array}{l}\text { Unterer Lias } \\
\text { (Hettang) }\end{array}$ & $\begin{array}{c}\text { Sondierung } 57 / 80 \\
\text { und } \\
\text { SB Babbenhausen } \\
1980\end{array}$ & 89340 & 85450 & $\begin{array}{l}14,0 \\
24,0\end{array}$ \\
\hline 6 & $\begin{array}{l}\text { Mühlenhof } \\
\text { Möllbergen }\end{array}$ & $\begin{array}{c}3719 \\
\text { Minden }\end{array}$ u. $\begin{array}{c}3819 \\
\text { Vlotho }\end{array}$ & 55 & 0,60 & 32 & $\begin{array}{l}\text { Saale } \\
\text { und } \\
\text { Elster }\end{array}$ & $\begin{array}{l}\text { Unterer Lias } \\
\text { (Hettang) }\end{array}$ & $\begin{array}{l}\text { SB Mühlenhof } \\
1995\end{array}$ & 94950 & 84700 & 38,0 \\
\hline 7 & $\begin{array}{c}\text { Nordbahnhof } \\
\text { Bad Oeynhausen }\end{array}$ & $\begin{array}{c}3718 \\
\text { Bad Oeynhausen }\end{array}$ & 56 & 0.12 & 28 & Holstein & Unterer Lias & $\begin{array}{l}\text { KB Nordbahnhof } \\
1994\end{array}$ & 86360 & 85740 & 32.0 \\
\hline 8 & Steinbeck-Loose & $\begin{array}{l}3818 \\
\text { Herford }\end{array}$ & 120 & 1.60 & 65 & $\begin{array}{c}\text { Saale } \\
\text { (Drenthe-Stadium) }\end{array}$ & Gipskeuper & $\begin{array}{c}\text { KB Steinbeck } \\
1976\end{array}$ & 84210 & 74220 & 67.0 \\
\hline 9 & $\begin{array}{c}\text { Ziegelei Bergmann } \\
\text { Hohenhausen }\end{array}$ & $\begin{array}{l}3819 \\
\text { Vlotho }\end{array}$ & 180 & 0,36 & 25 & $\begin{array}{l}\text { Weichsel } \\
\text { und } \\
\text { Eem }\end{array}$ & Gipskeuper & $\begin{array}{c}\text { Schappenbohrung } \\
\text { Bergmann } 1 \\
1989\end{array}$ & 95560 & 74260 & 18,0 \\
\hline 10 & $\begin{array}{c}\text { Großer Weserbogen } \\
\text { Vennebeck }\end{array}$ & $\begin{array}{c}3719 \\
\text { Minden }\end{array}$ & 45 & 0.76 & 43 & $\begin{array}{l}\text { Weichsel } \\
\text { und } \\
\text { Eem }\end{array}$ & $\begin{array}{l}\text { Unterer Lias } \\
\text { (Sinemur) }\end{array}$ & $\begin{array}{c}\text { KB/SB Großer } \\
\text { Weserbogen } \\
1987\end{array}$ & 89260 & 87610 & 48,0 \\
\hline
\end{tabular}

- Nummern wie in Abb. 2 und 3

$\mathrm{KB}=$ Kernbohrung

SB = Spülbohrung

\section{Das Untersuchungsgebiet und sein geologischer Bau}

Das Untersuchungsgebiet (Abb. 1) wird im Norden durch das Weser- und Wiehengebirge, im Süden durch den Teutoburger Wald begrenzt und umfaßt den Bereich von neun Blättern der Geologischen Karte von Nordrhein-Westfalen im Maßstab $1: 25000$. Es wird von einer mesozoischen Schichtenfolge Mittlerer Buntsandstein bis Oberer Jura aufgebaut, die ganz lokal von Schichten des Tertiärs, fast flächendeckend jedoch von Lockergesteinen des Quartärs überlagert wird. Zwischen 1911 und 1938 wurden diese von der Preußischen Geologischen Landesanstalt kartierten Blattgebiete in erster Auflage veröffentlicht. Damals fand der geologische Vorgang der Subrosion keinerlei Beachtung; heute sind etwa 70 Subrosionssenken bekannt, von denen zehn hier betrachtet werden sollen (Abb. 2, Tab. 1).
Voraussetzung für die Subrosion ist das Vorkommen auslaugungsfähiger Salinargesteine in ausreichender Mächtigkeit im tiefen Untergrund. Durch einige Tiefbohrungen im Untersuchungsgebiet (Abb. 2) und seiner näheren Umgebung wurden derartige Schichtenfolgen in vier stratigraphischen Einheiten nachgewiesen (KNAufF 1978, Deutloff 1995b, Farrenschon 1986, 1995b): Mittlerer Keuper: bis 25 m Anhydrit und Gips Mittlerer Muschelkalk: bis $20 \mathrm{~m}$ Anhydrit und Gips

Oberer Buntsandstein (Röt): bis $60 \mathrm{~m}$ Steinsalz, bis $40 \mathrm{~m}$ Anhydrit

Zechstein: bis $200 \mathrm{~m}$ Steinsalz, bis $100 \mathrm{~m}$ Anhydrit

Die äußeren Grenzen der Steinsalzverbreitung in den Schichten des Zechsteins und Röts verlaufen etwa parallel zum Nordrand der Rheinischen Masse durch das südliche Untersuchungsgebiet 
Tab. 2: Nachgewiesenes Pleistozän in Subrosionssenken Ostwestfalens.

Tab. 2: Proofed Pleistocene in subrosion depressions of Eastern Westphalia.

\begin{tabular}{|c|c|c|c|c|c|}
\hline $\begin{array}{c}\text { Jahre } \\
\text { vor } \\
\text { heute }\end{array}$ & \multicolumn{2}{|c|}{$\begin{array}{l}\text { Nordwest- } \\
\text { deutschland }\end{array}$} & \multicolumn{2}{|c|}{$\begin{array}{l}\text { Ostwestfalen } \\
\text { (Teilbereich) }\end{array}$} & $\begin{array}{l}\text { Nachweis } \\
\text { in } \\
\text { Subrosions- }\end{array}$ \\
\hline \multirow{3}{*}{-10.000} & \multirow{3}{*}{ Weichsel } & $\begin{array}{l}\text { Spät- } \\
\text { glazial }\end{array}$ & \multirow{3}{*}{$\begin{array}{c}\text { Weichsel } \\
\text { (w) }\end{array}$} & $\begin{array}{l}\text { Spät- } \\
\text { glazial }\end{array}$ & $\begin{array}{c}\text { Großer } \\
\text { Weserbogen }\end{array}$ \\
\hline & & $\begin{array}{l}\text { Hoch- } \\
\text { glazial }\end{array}$ & & $\begin{array}{l}\text { Hoch- } \\
\text { glazial }\end{array}$ & $\begin{array}{c}\text { Schlachthof } \\
\text { Bad Oeynhausen }\end{array}$ \\
\hline & & $\begin{array}{l}\text { Früh- } \\
\text { glazial }\end{array}$ & & $\begin{array}{l}\text { Früh- } \\
\text { glazial }\end{array}$ & $\begin{array}{c}\text { Blutwiese } \\
\text { Gohfeld }\end{array}$ \\
\hline-110000 & \multicolumn{2}{|c|}{ Eem } & \multicolumn{2}{|c|}{$\begin{array}{l}\text { Eem } \\
\text { (ee) }\end{array}$} & $\begin{array}{c}\text { Ziegelei } \\
\text { Bergmann } \\
\text { Großer } \\
\text { Weserbogen }\end{array}$ \\
\hline \multirow{4}{*}{-127000} & \multirow{3}{*}{ Saale } & Warthe & \multirow{3}{*}{$\begin{array}{l}\text { Saale } \\
\text { (sa) }\end{array}$} & n.n. & \\
\hline & & Vorselaer & & n.n. & \\
\hline & & Drenthe & & $\begin{array}{l}\text { Drenthe } \\
\text { (D) }\end{array}$ & Steinbeck-Loose \\
\hline & \multicolumn{2}{|c|}{ Holstein } & \multicolumn{2}{|c|}{$\begin{array}{l}\text { Holstein } \\
\text { (ho) }\end{array}$} & $\begin{array}{c}\text { Nordbahnhof } \\
\text { Bad Oeynhausen } \\
\text { Iggenhausen }\end{array}$ \\
\hline-330000 & \multicolumn{2}{|c|}{ Elster } & \multicolumn{2}{|c|}{$\begin{array}{c}\text { Elster? } \\
(\mathrm{e} ?)\end{array}$} & $\begin{array}{l}\text { Mühlenhof } \\
\text { Möllbergen }\end{array}$ \\
\hline \multirow[t]{3}{*}{790000} & \multicolumn{2}{|c|}{ Cromer } & \multicolumn{2}{|c|}{$\begin{array}{l}\text { Cromer } \\
\text { (cr) }\end{array}$} & $\begin{array}{c}\text { Babbenhausen } \\
\text { Exter-Krutheide } \\
\text { Mosebeck }\end{array}$ \\
\hline & \multicolumn{2}{|c|}{ Bavel } & \multicolumn{2}{|c|}{$\begin{array}{l}\text { Bavel } \\
\text { (bv) }\end{array}$} & $\begin{array}{c}\text { Mosebeck } \\
\text { Exter-Krutheide } \\
\text { Schlachthof } \\
\text { Bad Oeynhausen }\end{array}$ \\
\hline & & & $\begin{array}{r}\mathrm{Mer} \\
\text { (m }\end{array}$ & & Mosebeck \\
\hline \multirow[t]{4}{*}{930000} & \multicolumn{2}{|c|}{ Waal } & \multicolumn{2}{|c|}{$\begin{array}{l}\text { Waal } \\
\text { (wa) }\end{array}$} & Mosebeck \\
\hline & \multicolumn{2}{|c|}{ Eburon } & \multicolumn{2}{|c|}{ n.n. } & \\
\hline & \multicolumn{2}{|c|}{ Tegelen } & \multicolumn{2}{|c|}{ n.n. } & \\
\hline & \multicolumn{2}{|c|}{ Prätegelen } & \multicolumn{2}{|c|}{ n.n. } & \\
\hline
\end{tabular}

und begrenzen dadurch im wesentlichen das Gebiet möglicher Subrosion (Abb. 2). Der geologische Vorgang der Subrosion von Steinsalz, Anhydrit und Gips im Untergrund durch zirkulierende vadose oder auch juvenile Grundwässer wird hier als bekannt vorausgesetzt. Durch juvenile, $\mathrm{CO}_{2^{-}}$ reiche Tiefenwässer als Endausscheidungen des jungtertiären nordhessischen Basaltvulkanismus soll die Subrosion verstärkt worden sein, beispielsweise im Raum Bad Pyrmont (Hermann
1968). Der Senkungsvorgang vermag sich durch hunderte Meter mächtige Gesteinspakete bis zur Erdoberfläche durchzupausen (HERmann 1972 ).

\section{Das Pleistozän Ostwestfalens}

\section{1 Übersicht}

Die geologischen Hauptereignisse während des Pleistozäns waren im mitteleuropäischen Raum drei Vorstöße des skandinavischen Inlandeises in den Kaltzeiten Elster, Saale und Weichsel, welche durch Holstein- und Eem-Warmzeit voneinander getrennt sind. Dem Elster gingen mehrere Kaltund Warmzeiten voraus, deren Ablagerungen am vollständigsten aus dem Mündungsgebiet von Rhein und Maas in den Niederlanden (ZAGWIJN 1985) sowie aus der Subrosionssenke über dem Satzstock Lieth in Schleswig-Holstein (MENKE 1975, Stephan \& Menke 1994) überliefert sind. Gleichaltrige Bildungen blieben unter günstigen Bedingungen aber auch im nordwest- und mitteldeutschen Flachland sowie in den südlich anschließenden Mittelgebirgslandschaften in unterpleistozänen Entwässerungsrinnen und besonders in Subrosionssenken über den dortigen Salinargesteinsfolgen erhalten.

Die Gesamtgliederung des Pleistozäns in Nordrhein-Westfalen und die bisher in Ostwestfalen nachgewiesenen stratigraphischen Einheiten sind in Tab. 2 zusammengefaßt. Im folgenden werden diese Einheiten nach Gesteinszusammensetzung und Polleninhalt in chronologischer Reihenfolge beschrieben.

\subsection{Unterpleistozän}

Das Unterpleistozän Ostwestfalens wurde vor allem durch die Bohrung Mosebeck 1976 des Geologischen Landesamts Nordrhein-Westfalen in der gleichnamigen Subrosionssenke (TK 25 Blatt 4019 Detmold) erschlossen (Abb. 3 Nr. 1). Die Bohrung wurde von O. Deutloff lithologisch aufgenommen, von H.-W. ReHAGEN erstmals pollenstratigraphisch untersucht (REHAGEN 1980) und in den Erläuterungen zur geologischen Neuaufnahme des Blattes Detmold publiziert (FARrENSCHON 1986: 147). Dabei stellte ReHAGEN (1980) den überwiegenden Anteil der erbohrten Abfolge (57 - $134 \mathrm{~m}$ Teufe) in das Waal, Menap und Bavel sensu ZaGWIJN $(1957,1963)$ des Unterpleistozäns. Eine eindeutige Parallelisierung mit den Typlokalitäten gelang jedoch nicht. Daher wird das Pleistozän der Bohrung Mosebeck 1976 derzeit überarbeitet; detaillierte Ergebnisse sind dabei einer späteren Publikation vorbehalten. 
Das Unterpleistozän der Bohrung Mosebeck soll hier lediglich am Beispiel einer $15 \mathrm{~m}$ mächtigen Abfolge exemplarisch dargestellt werden, die ReHagen in das Bavel stellt (Abb. 4). Zunächst ist eine Picea-Tenga-Phase überliefert, in der Betula nur eine untergeordnete Rolle spielt. Die PiceaQuote geht im Hangenden zugunsten thermophiler Gehölze zurück. Das Klimaoptimum der Warmzeit ist durch einen ausgeprägten Eichenmischwald mit Quercus, Ulmus, Tilia und Fraxinus sowie ausgesprochen hohe Carpinus- und vor allem Tsuga-Werte gekennzeichnet. Die Eucommia-Kurve ist auf niedrigem Niveau nahezu geschlossen. Die Warmzeit endet mit einer borealen Phase, die durch Pinus und Betula bestimmt wird.

Im Jahr 1980 fand REHAGEN ähnliche pollenfloristische Verhältnisse auch in der Subrosionssenke von Exter-Krutheide in einer Bohrung für die Neukartierung des Blattes 3818 Herford vor (Deutloff 1995b: 71), ferner in der Kernbohrung 10/A30 1985 (Abb. 3 Nr. 2) für die Autobahnplanung in der Subrosionssenke Schlachthof Bad Oeynhausen, Blatt 3718 Bad Oeynhausen (ReHAGEN, unveröff. Ber.).

Die Abschwemm-Massen sind in den drei Subrosionssenken petrographisch recht mannigfaltig zusammengesetzt: Die Korngrößen reichen von Ton über Silt - teilweise humos mit einzelnen Torflagen - bis zu Sand und Kies.

In der Subrosionssenke von Exter-Krutheide lieferte die Baugrundbohrung Kläranlage VlothoExter aus 5,7 - 7,0 m Teufe ein Pollenspektrum mit markanten Anteilen der jungtertiären bis unterpleistozänen Gattungen Tsuga und Ostrya, während die Kartierbohrung 10/79 Pahmeier aus 14 - 18 m Teufe eine Pollenassoziation mit geringen, aber typischen Anteilen von Tsuga, Eucommia und Pterocarya ergab.

In der Subrosionssenke Schlachthof Bad Oeynhausen lieferte die Kernbohrung 10/A30 1985 aus dem pollenführenden Bereich von 16,3 - 18,8 m Teufe ein Pollenspektrum mit Pterocarya, Tsuga, Carya, Eucommia und Ostrya. Hier sind Carpinus-Tsuga-Vergesellschaftungszonen wie in der Bohrung Mosebeck typisch.

\subsection{Cromer-Komplex}

Das Cromer umfaßt sechs Warm- und fünf Kaltzeiten (CASPERs et al. 1995: Tab. 1). Für das südniedersächsische Nachbargebiet Ostwestfalens gibt die Quartärgeologische Übersichtskarte von Niedersachsen und Bremen 1:500000 (Nieder-
sächs.L.-Amt Bodenforsch. 1995) sechs pollenanalytisch datierte Vorkommen von Teilbereichen des Cromers an, wovon die meisten gleichfalls in Subrosionssenken gelegen sind. So wurde im Auslaugungsbereich des Münder-Mergel-Salinars (Oberer Malm) im Blattgebiet 3515 Hunteburg nordöstlich Osnabrück durch eine Forschungsbohrung des Niedersächsischen Landesamts für Bodenforschung die frühcromerzeitliche „Hunteburg-Warmzeit" pollenanalytisch nachgewiesen (HAHNE et al. 1994: 117).

Auch in Ostwestfalen fanden sich cromerzeitliche Ablagerungen in Subrosionssenken, so z. B. bei der Neukartierung der Blattgebiete 4020 Blomberg (FARRENSCHON 1995b: 86) und 3919 Lemgo (FARRENSCHON 1998). Im Stadtgebiet Blomberg liegt der unter Quartär-Geologen altbekannte Aufschluß der noch heute betriebenen Ziegeleitongrube Hohedömsen (Abb. 2 Nr. 12) mit humosen, tonig-siltigen Abschwemm-Massen des Cromers (ReHAGEN 1980: 61; FARRENSCHON in Schirmer 1995a: 586; FARRENSCHON 1995b: 126).

In der Subrosionssenke von Babbenhausen im Stadtgebiet Bad Oeynhausen wurde Ende der 70er Jahre beim Bau des dortigen Autobahnkreuzes ein über $30 \mathrm{~m}$ mächtiges Pleistozän-Profil aufgeschlossen, das hauptsächlich der Saale-Zeit zuzuordnen ist (siehe unten). Unter der Sohle des zeitweiligen Aufschlusses wurden durch die Sondierbohrung 57/80 des Geologischen Landesamts NRW rund 9 m schwarzgrauer, zäher, kalkfreier, humoser, siltiger Ton mit dünnen Lagen von Feinund Mittelkies aus Keuper-Gesteinen erschlossen, durch die dicht benachbarte Spülbohrung Babbenhausen 1980 (Abb. 3 Nr. 5) das Gesamtprofil bis zum liegenden Lias-Tonstein.

Das Pollendiagramm (Abb. 5) läßt eine Warmzeit erkennen, deren Beginn nicht überliefert ist, da das Profil mit ausgeprägten Anteilen des EMW beginnt: Quercus, vor allem aber Ulmus und Tilia finden sich in größeren Anteilen. Neben diesen Gehölzen belegen Hedera und Ilex ein warmes, feuchtes Klima. Nachfolgend werden die Wälder vornehmlich von Carpinus und Pterocarya gebildet. Auch Taxus, Larix und Tsuga sind deutlich vertreten. In den oberen Partien des untersuchten Abschnitts endet der Nachweis von Hedera und Ilex. Pinus und Picea werden bedeutsamer. Das Klima wird kontinentaler. Auffallend ist ein kurzes Maximum (>10\%) von Abies, die offenbar $\mathrm{Ul}$ mus, Tilia und Acer zurückdrängte, da deren Kurven vorher enden. Die Kurve von Abies endet dann gemeinsam mit denen von Pterocarya, Car- 


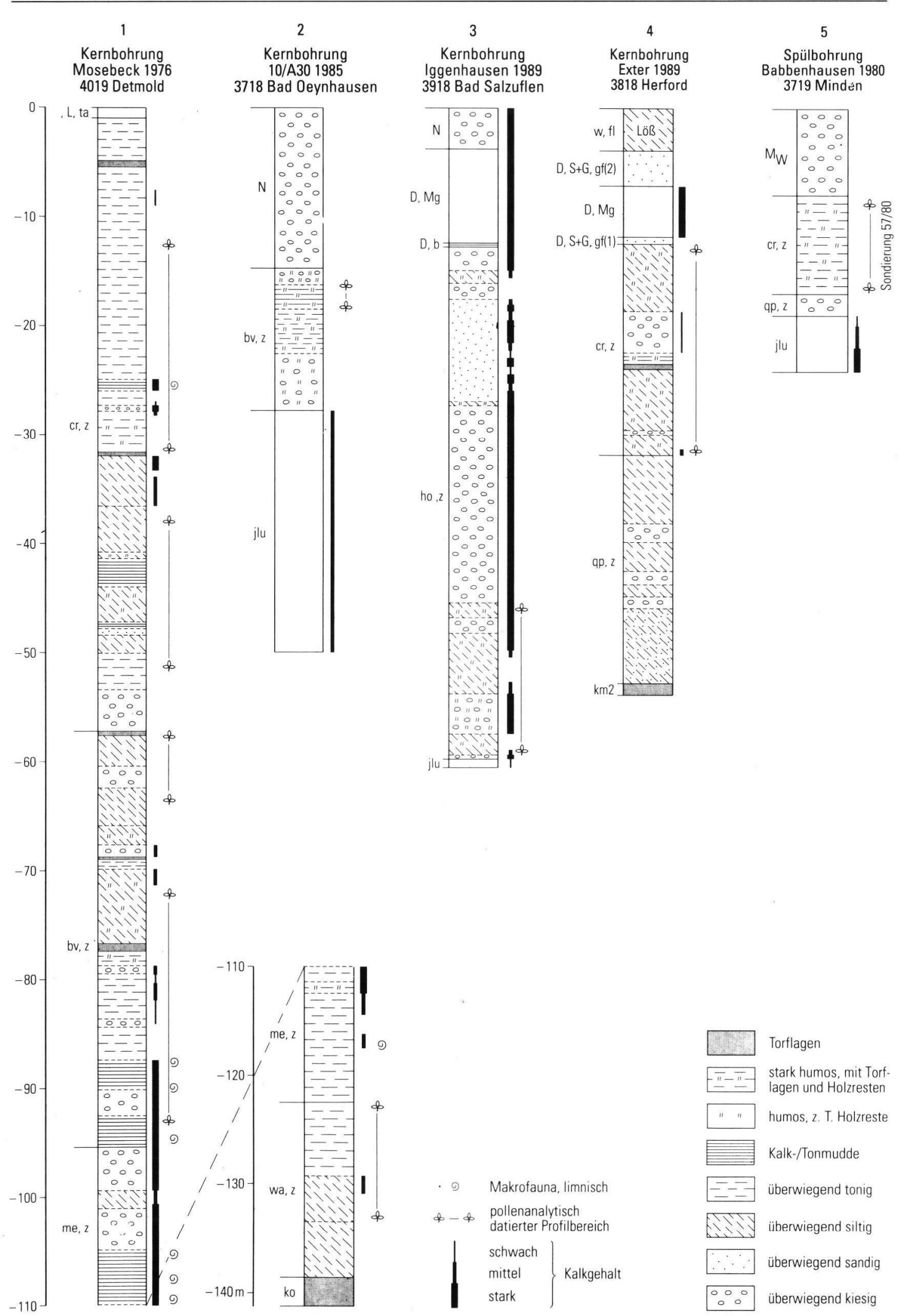


6

Spülbohrung Mühlenhof 1995

3819 Vlotho

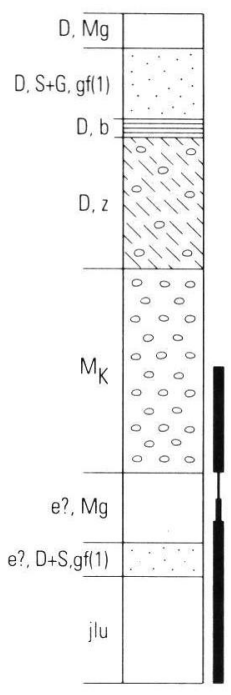

L, ta Auenlehm

W, fl Fließerde

N Niederterrasse

ee, 2 Eem-Schichten

$D, f \mid \quad F l i e ß e r d e$

D, S+G, gf(2) Nachschüttsand

$D, K$ und Kame

$\mathrm{D}, \mathrm{Mg}$ Grundmoräne

D,S+G, gf(1) Vorschüttsand

D, b $\quad \begin{array}{ll}\text { Beckenton und } \\ \text { Beckenschluff }\end{array}$

D, z Abschwemmassen

$M_{W} \cdot M_{K} \quad$ Mittelterrasse

W. M (Ur-Werre, Ur-Kalle)

ho, $z$ Holstein-Schichten

$e$ ?, Mg Grundmoräne

e?, $S+G, g f(1) \quad$ Vorschüttsand

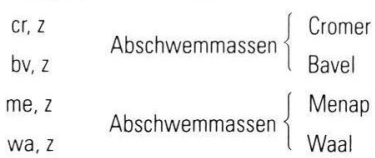

Abschwemmassen,

$q p, z \quad$ nicht datiert

jlu Lias, unterer

ko, km(1-2) Keuper, Oberer und Mittlerer
7

Kernbohrung Nordbahnhof 1994 3718 Bad0eynhausen
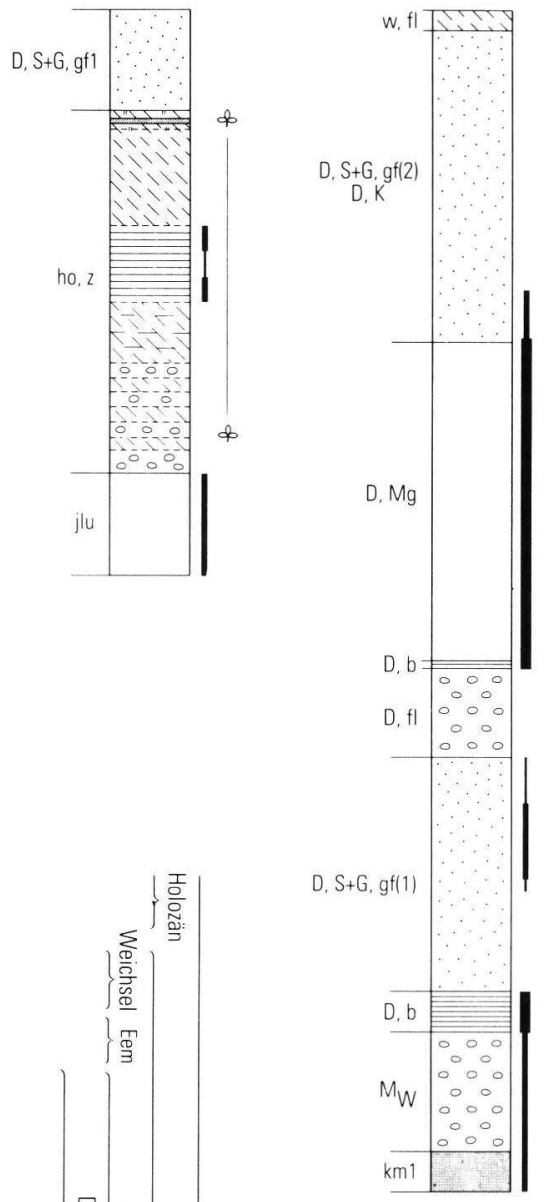

Kernbohrung Steinbeck 1976 3818 Herford

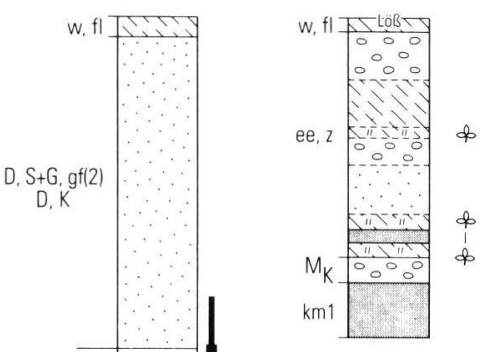

9

10

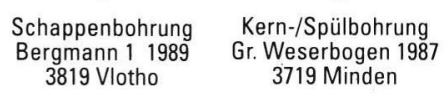

3719 Minden
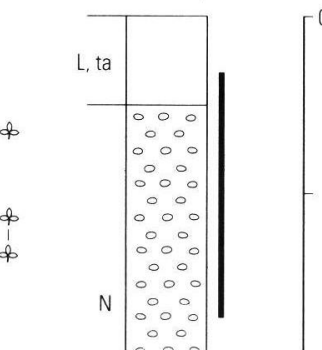

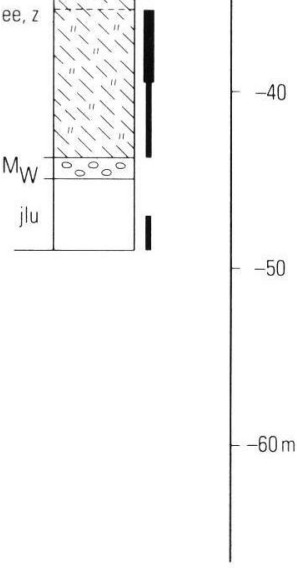

Abb. 3: Geologie und Lithostratigraphie der untersuchten Bohrungen.

Fig. 3: Geology and lithostratigraphy of the wells investigated. 


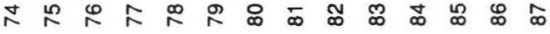
mाmा

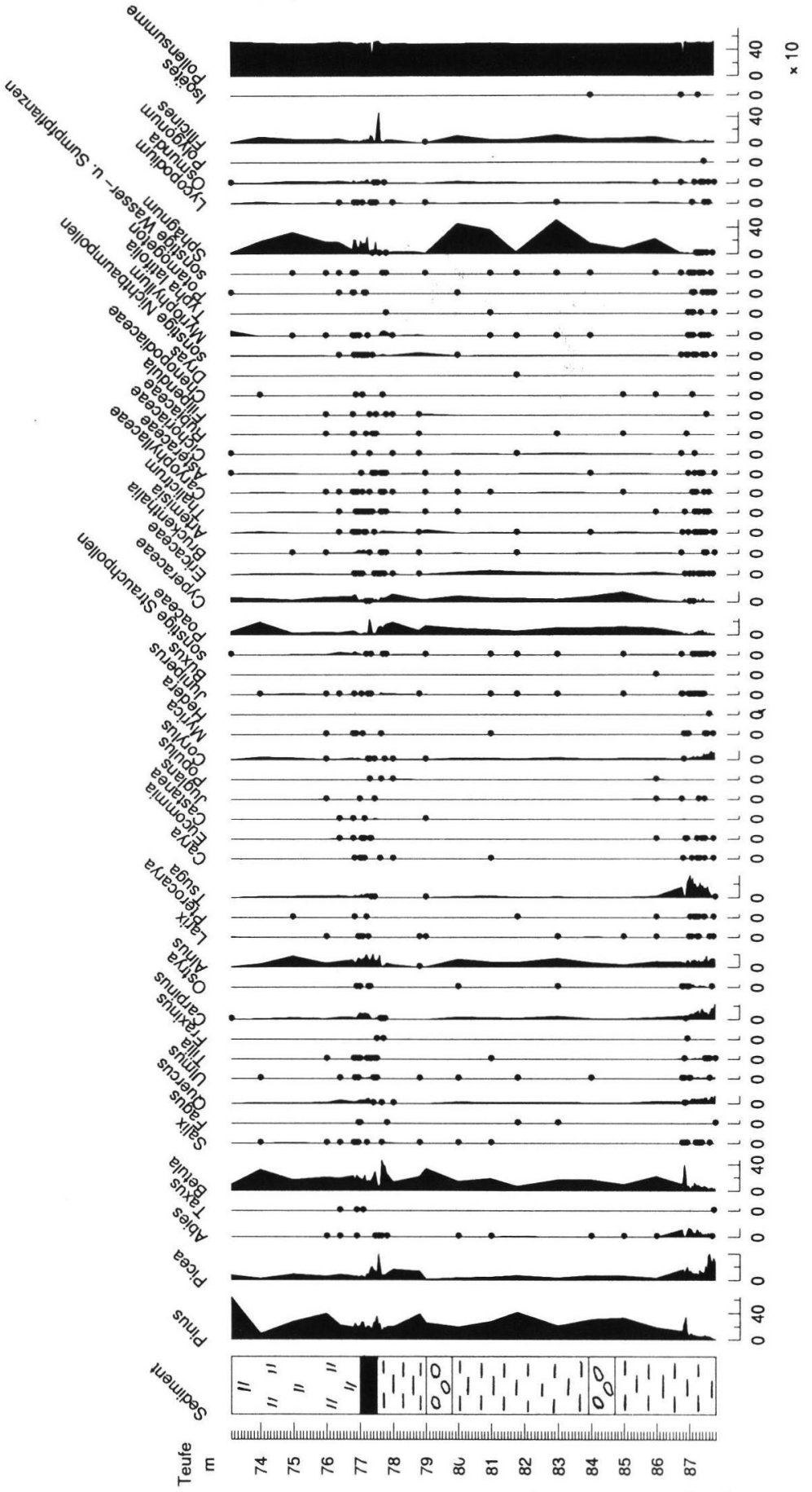

Abb. 4: Pollendiagramm eines unterpleistozänen Abschnitts in der Bohrung Mosebeck. Schwarze Punkte markieren Pollenpro zente unter $2 \%$.

Fig. 4: Pollen diagram of lower Pleistocene age in the well Mosebeck. Black dots indicate pollen percentages below $2 \%$. pinus, Quercus und Ulmus. Dies belegt wohl einen deutlichen Einschnitt im Klima- und Vegetationsablauf: Das Klimaoptimum ist überschritten. Die Bildung borealer Nadelwälder wird begünstigt. Das Ende der Warmzeit markieren die Anstiege der Betula- und Gramineen-Kurven. Die Pinus-Quoten werden stetig geringer. Es bildet sich die Tundrenvegetation eines Stadials, das jedoch nicht durch hangende Moränensedimente belegt wird, da das Profil eine Schichtlücke bis zur frühsaalezeitlichen Mittelterrasse aufweist. Eine Parallelisierung mit pleistozänen Pollenprofilen andernorts ist auch hier schwierig. Sicher ist zunächst das unterpleistozäne Alter aufgrund der deutlichen Anteile unterpleistozäner Gattungen, insbesondere Tsuga und Pterocarya. Derartig deutliche Tsuga-Anteile wie in Bad Oeynhausen-Babbenhausen sind sonst nur aus vorcromerzeitlichen Profilen bekannt, doch lassen sich die hier vorgefundenen Verhältnisse nicht mit entsprechenden Abschnitten der Bohrung Mosebeck parallelisieren, wo das Unterpleistozän zweifelsfrei vorhanden ist. Insbesondere fehlt in $\mathrm{Bad}$ Oeynhausen-Babbenhausen eine ausgeprägte Carpinus-Tsuga-Zone, zum anderen erreicht Pterocarya hier weitaus höhere Werte.

Abgesehen davon stimmen Details dieses Profils mit cromerzeitlichen Profilen der weiteren Umgebung, etwa Hunteburg bei Osnabrück (HAHNE et al. 1994) oder Sohlingen im Solling (HOMANN \& LePPER 1994), überein. So spielt Abies eine beträchtliche Rolle; Taxus ist gut vertreten. Der EMW ist deutlich repräsentiert; insbesondere Tilia erreicht relativ hohe Werte. Ulmus breitet sich relativ früh, Corylus hingegen gegenüber dem EMW verzögert aus.

In der bei Deutloff (1995b: 71, 160) lithologisch ausführlich beschriebenen Rammkernbohrung 


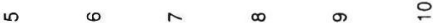

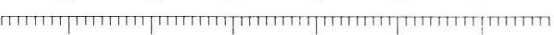
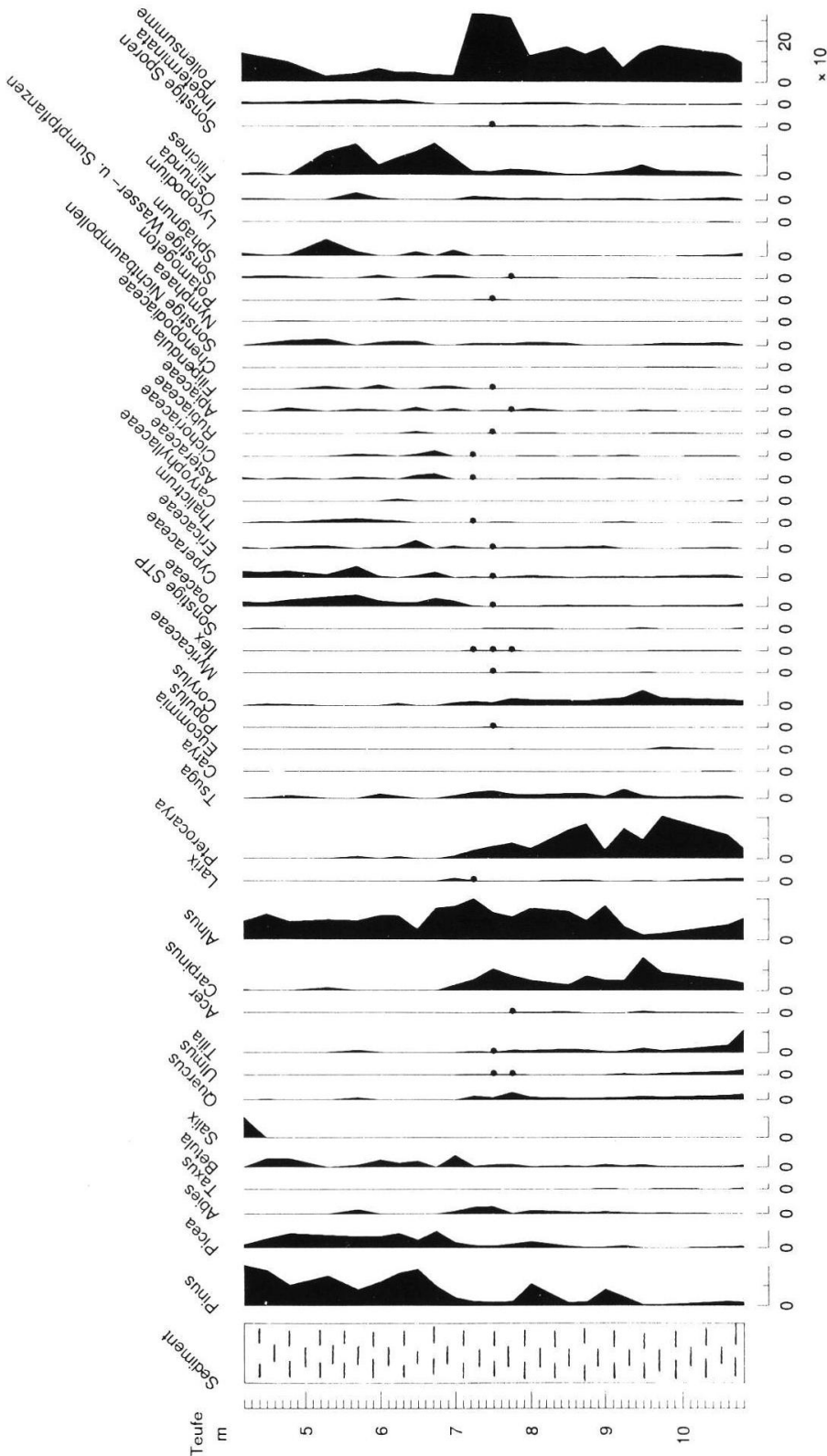

Abb. 5: Pollendiagramm des cromerzeitlichen Abschnitts in der Bohrung Babbenhausen. Schwarze Punkte markieren Pollenprozente unter 2\%. Weitere Legende siehe Abb. 3.

Fig. 5: Pollen diagram of Cromerian age in the well Babbenhausen.

Black dots indicate pollen percentages below $2 \%$.
Exter 1989 in Vlotho-Exter (Abb. 3 Nr. 4) enthielt nur die obere Hälfte der 40,5 m mächtigen AbschwemmMassen bestimmbare Pollenfloren. Neben erkennbar umgelagerten Palynomorphen des Frühtertiärs läßt sich ein parautochthones Pollenspektrum erkennen, das von Pinus und Picea beherrscht wird (Abb. 6). Gemeinsam mit deutlichen Betula- und Salix-Quoten wird ein weitgehend geschlossener (BP:NBP > 1), von winterharten Gehölzen bestimmter Wald dokumentiert. Thermophile Gattungen sind unbedeutend. So sind nur die Kurven von Quercus und Carpinus etappenweise auf niedrigem $\mathrm{Ni}$ veau geschlossen. Auffällig ist eine sippenreiche, jungtertiäre/altpleistozäne Flora mit Larix, Eucommia, Pterocarya und Tsuga, die sich über das ganze Profil verteilt findet.

Aufgrund der geologischen Position ist nur ein präsaalezeitliches Alter möglich. Dabei schränken paläomagnetische Messungen an den Bohrkernen (ZuBRÄGEL 1991) das Alter auf den Cromer-Komplex ein: In $23 \mathrm{~m}$ Bohrtiefe wurde die Brunhes/Matujama-Grenze (790 000 Jahre vor heute) nachgewiesen, die nach ZAGWIJN (1985) im unteren Cromer (Cromer I) liegt. Allerdings wurde bereits $3 \mathrm{~m}$ tiefer der Jaramillo-Event (930 000 Jahre vor heute) gemessen, der von ZAGWIJN in das Bavel gestellt wurde (zur Abstimmungsproblematik zwischen paläomagnetischer und pollenanalytischer Datierung siehe Fromm 1994: 252). Das Bavel scheint aber in Ostwestfalen-Lippe durch wesentlich höhere TsugaWerte gekennzeichnet zu sein, so daß der beschriebene Abschnitt der Bohrung Exter in das untere Cromer gehören dürfte. Die nächsten vergleichbaren Fundorte dieses Alters liegen bei Osnabrück (Hunteburg GE 58, HAHNE et al. 1994) und im Solling (Sohlingen, Homann \& Lepper 1994). Vergleiche 


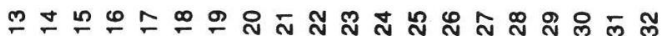

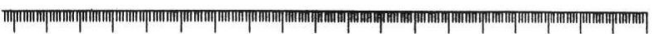

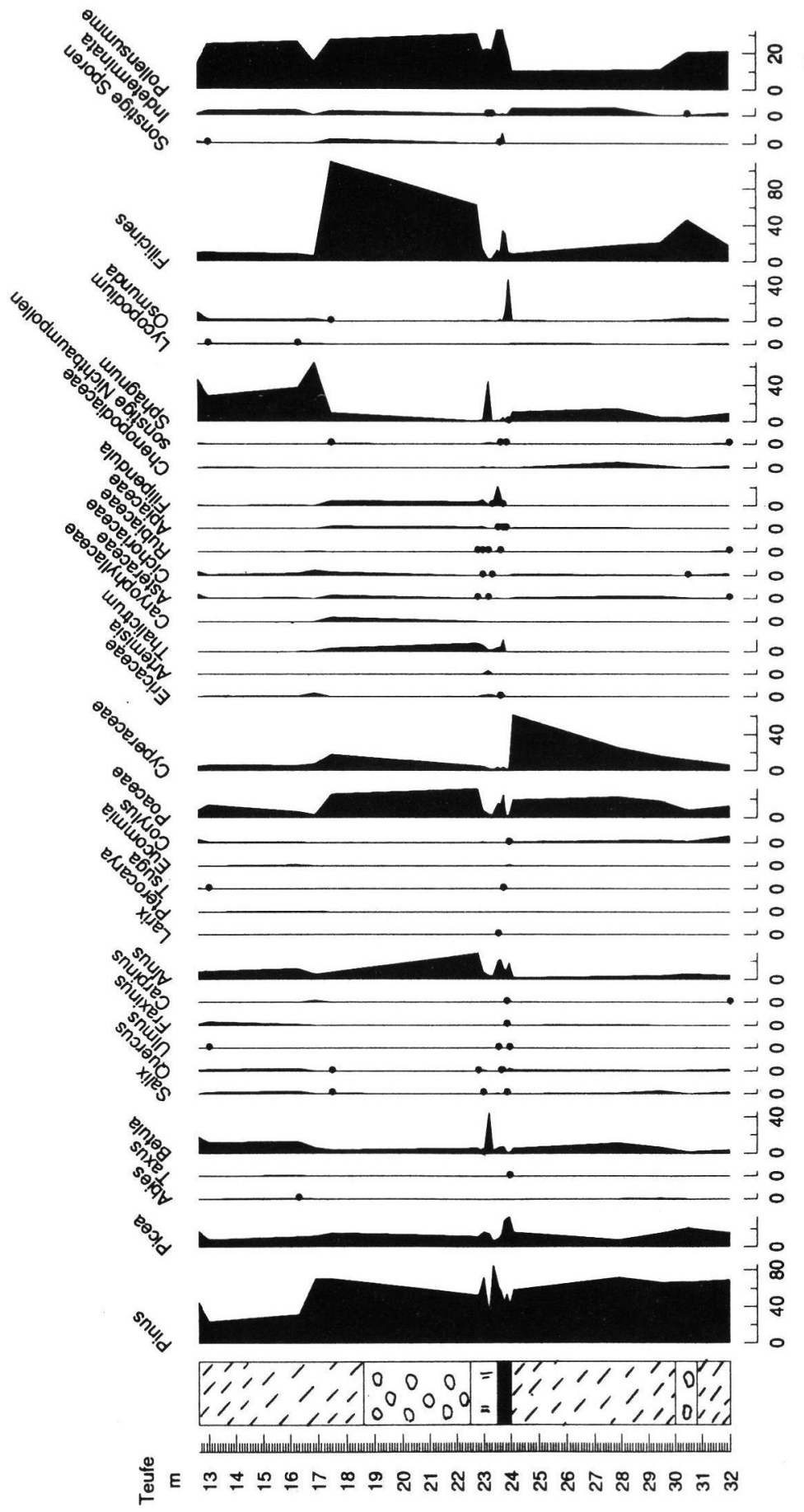

mit ihnen sind auch deshalb schwierig, da der untersuchte Abschnitt der Bohrung Exter nur einen früh- oder spätwärmezeitlichen Abschnitt repräsentiert. Mögliche Parallelen ergeben sich im Vergleich mit höheren Abschnitten des Profils in Sohlingen, die sich ebenso durch dominierende winterharte Gehölze und geringe Anteile thermophiler Gattungen sowie von Eucommia und Pterocarya auszeichnen.

\subsection{Elster-Kaltzeit}

Die bisher beschriebenen waal- bis cromerzeitlichen Sedimentfüllungen von Subrosionssenken dokumentieren ganz lokale geologische Vorgänge innerhalb der pleistozänen Entwicklungsgeschichte des Weserberglandes. Zu Beginn des Elsters veränderte dann der erste Inlandeisvorstoß aus Skandinavien die mitteleuropäische Landschaft grundlegend, indem flächenhaft Staubecken-, Schmelzwasser- und Gletscherschuttsedimente abgelagert wurden.

Aus dem mitteldeutschen Hauptverbreitungsgebiet mit seinen ausgedehnten Tagebauaufschlüssen liegen darüber moderne zusammenfassende Darstellungen von EISSMANN (1994) und KNOTH (1995) vor. Für die vorliegende Arbeit ist der dortige Befund wichtig, daß die elsterzeitlichen Grundmoränen außer in Rinnen auch in Subrosionssenken Mächtigkeiten bis 70 m erreichen (KNOTH 1995: 154). Östlich des Untersuchungsgebietes verläuft in Südniedersachsen gemäß der genannten Übersichtskarte die Außengrenze der ElsterVereisung vom Leinebergland nördlich Northeim (Subrosionssenke von Ahlshausen (JORDAN \& SCHWARTAU 1993) durch das Wesertal zwischen Bodenwerder und $\mathrm{Ha}$ meln (Weserlaufverlegung, ROHDE 1994) in das Emmertal bis zur Landesgrenze bei Lügde. Diese Aussage widerspricht den Erkenntnissen

Abb. 6: Pollendiagramm der Bohrung Exter. Schwarze Punkte markieren Pollenprozente unter $2 \%$.

Fig. 6: Pollen diagram of the well Exter. Black dots indicate pollen percentages below $2 \%$. 


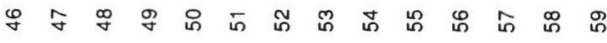

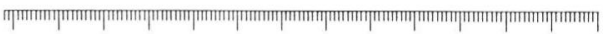

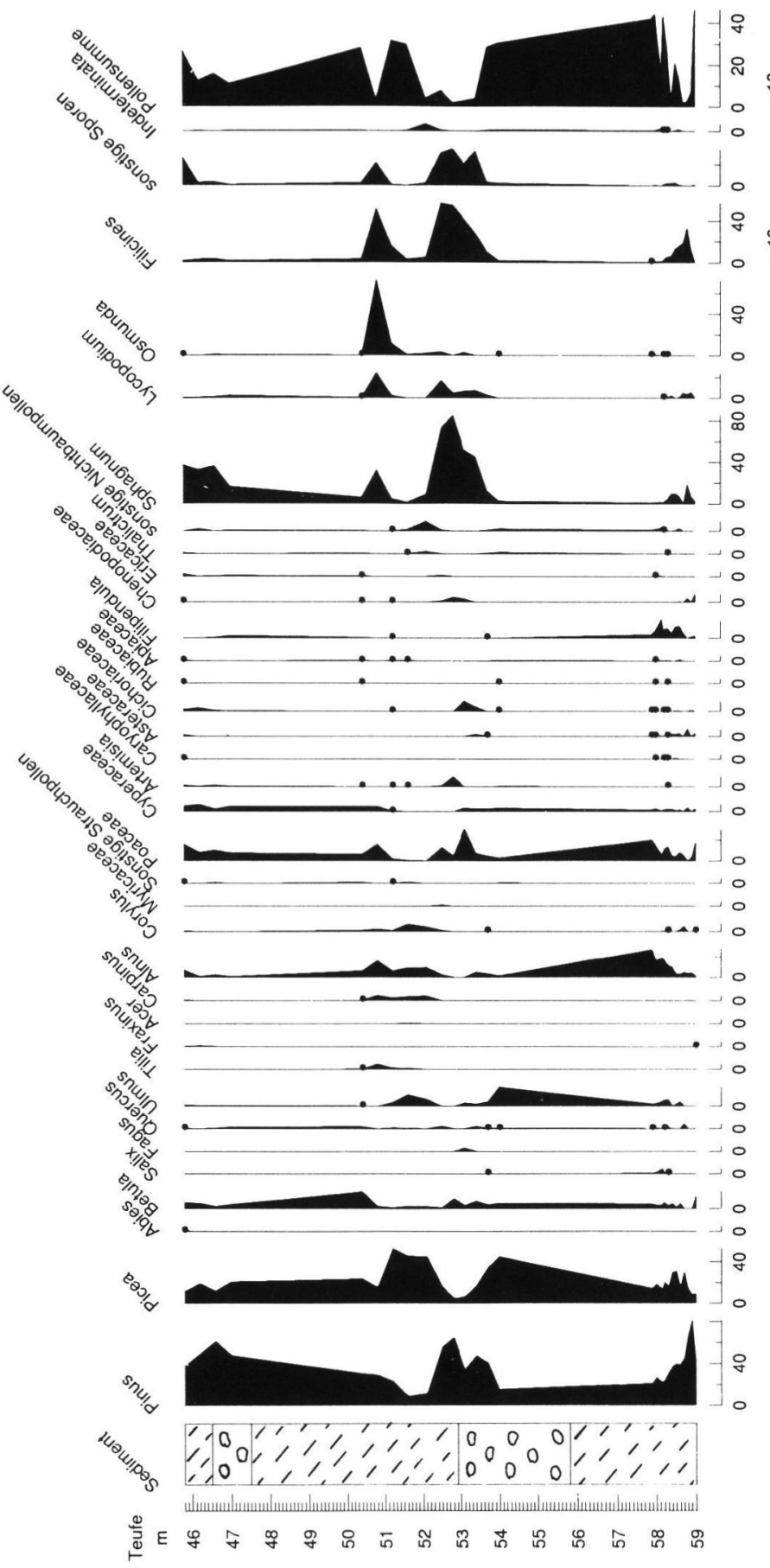

Abb. 7: Pollendiagramm eines holsteinzeitlichen Abschnitts in der Bohrung Iggenhausen. Schwarze Punkte markieren Pollenprozente unter $2 \%$.

Fig. 7: Pollen diagram of Holsteinian age in the well Iggenhausen. Black dots indicate pollen percentages below $2 \%$. von Skupin, Speetzen \& Zandstra (1993: 68), wonach in NordrheinWestfalen stratigraphisch gesicherte elsterzeitliche Moränenvorkommen fehlen.

× Möglicherweise könnten sich im Verbreitungsgebiet der saalezeitlichen Vereisung südlich des Weserund Wiehengebirges (Abb. 2) glazigene Gesteine des Elsters nicht oberflächlich, aber in den Sedimentfallen der Subrosionssenken erhalten haben. Allerdings blieb trotz intensiver Bohrtätigkeit bis 1995 der Nachweis elsterzeitlichen Geschiebemergels auf nicht eindeutige Befunde in Subrosionssenken des Blattgebietes $3918 \mathrm{Bad}$ Salzuflen beschränkt (JäGER in Knauff 1978: 56, Deutloff 1995b: 73).

Im Jahre 1995 wurden in der Subrosionssenke Mühlenhof Möllbergen (Blattgebiete 3719 Minden und 3819 Vlotho) rund $6 \mathrm{~km}$ südlich der Porta Westfalica durch die Spülbohrung Mühlenhof 1995 (Abb. 3 Nr. 6) zwei Geschiebemergel erschlossen, die durch $23 \mathrm{~m}$ Sediment voneinander getrennt werden. Der obere entkalkte Geschiebemergel wird von Vorschüttkiessand, Beckenton und $7 \mathrm{~m}$ mächtigen, siltigen Abschwemm-Massen unterlagert, die dem Drenthe-Stadium zugeordnet werden können. Im Liegenden folgt ein $11 \mathrm{~m}$ mächtiger, kalkhaltiger, bunter Kiessand, der Muschelkalk-Gerölle des wenige $\mathrm{km}$ südlich gelegenen Kalldorfer Sattels enthält. Er ist außerhalb der Subrosionssenke in einer Bachuferböschung aufgeschlossen und wurde von Rӧнм (1985) als Mittelterrassenkörper einer „UrKalle" identifiziert, die von Süden her dem damaligen Weserlauf zufloß.

Der unterlagernde untere Geschiebemergel ist ein $4 \mathrm{~m}$ mächtiger hell- bis dunkelgrauer, nach unten zunehmend kalkreicherer stark sandiger, toniger Silt mit nordischen Geschieben. Bis zur Quartär- 
Basis folgt ein $2 \mathrm{~m}$ mächtiger, dunkelgrauer, stark kalkhaltiger Vorschüttkiessand, der ebenfalls nordisches Material enthält. Nach den Kriterien von KalTwang (1992: 13) ist damit der untere Geschiebemergel als Grundmoräne des Elsters anzusprechen. Dieser vermutlich erste in-situ-Nachweis einer elsterzeitlichen Grundmoräne südlich der Porta Westfalica bedarf sicherlich ergänzender Untersuchungen (Kernbohrungen).

In dieses Bohrergebnis fügt sich der Fund einer isolierten Geschiebemergel-Scholle mit einer für das Elster typischen Geschiebezusammensetzung in der drenthezeitlichen Kame-Terrasse bei Möllenbeck (Blatt 3820 Rinteln, R 01300, H 81000) ein (Wellmann 1990).

\subsection{Holstein-Warmzeit}

Nach dem Ende des Elsters wurden in einigen Subrosionssenken Ostwestfalens erneut humose, pollenführende, siltig-tonige Sedimente abgelagert. Einen ersten palynologischen Nachweis des Holsteins lieferte bei der Neubearbeitung des Blattes 3918 Bad Salzuflen die Kernbohrung Holzhausen 1970 des Geologischen Landesamts NRW in der gleichnamigen Subrosionssenke (Abb. 2 Nr. 13) (Pollenanalyse durch H.-W. ReHAGEN). Sie erschloß eine $15,5 \mathrm{~m}$ mächtige Wechselfolge von Niedermoortorf, Tongyttja, dunkelgrauem humosem Ton und Kiessand (KNauff 1978: 57,119).

In der Subrosionssenke Iggenhausener Wald in Lage-Pottenhausen durchteufte die Rammkernbohrung Iggenhausen 1989 (Abb. $3 \mathrm{Nr}$. 3) unter der rund $8 \mathrm{~m}$ mächtigen Grundmoräne des Drenthe-Stadiums der Saale-Kaltzeit $47,7 \mathrm{~m}$ Abschwemm-Massen. Diese bestehen zu über $60 \%$ aus kalkhaltigem, stark tonig-sandigem Fein- und Mittelkies mit einem Geröllbestand aus Teutoburger-Wald-Gesteinen ohne nordische Komponente. Aus den eingelagerten, stark humosen Silten und Tonen zwischen 44 und $58 \mathrm{~m}$ Teufe konnte eine Pollenflora isoliert werden, die gleichförmig von Pinus und Alnus beherrscht wird (Abb. 7). Picea zeigt ein ausgesprochenes Maximum. Der EMW ist bis auf Quercus, deren Kurve fast geschlossen ist, bedeutungslos. Ebenso verhält es sich mit Corylus, Abies und Carpinus. Diese Charakteristika sprechen für eine Einstufung in die Holstein-Warmzeit. Die vorgefundenen Gegebenheiten lassen sich gut mit denen des Profils Munster-Breloh in der Lüneburger Heide (H. MüLLER 1974) vergleichen.

In der Subrosionssenke Nordbahnhof Bad Oeynhausen, Blatt 3718 Bad Oeynhausen, lieferten
1983 ein Baugrubenaufschluß und 1994 die ingenieurgeologische Kernbohrung Nordbahnhof 1994 (Abb. 3 Nr. 7) ein gesichertes pollenstratigraphisches Profil dieser Warmzeit. Die Bohrung durchteufte unter Vorschüttsand des Drenthe-Stadiums der Saale-Kaltzeit rund $18 \mathrm{~m}$ humosen Silt, in den geringmächtige Feinsande und Tone eingeschaltet sind. Der palynologische Befund spricht für eine Einstufung in das Holstein. Neben dominierenden Kiefern- und Birkenanteilen sind vor allem hohe Haselanteile sowie der Nachweis jungtertiärer Florenelemente wie Pterocarya und Nyssa markant.

\subsection{Saale-Kaltzeit}

In der Saale-Kaltzeit kam es zu mindestens zwei Vorstößen des nordeuropäischen Inlandeises während des Drenthe- und des Warthe-Stadiums. Diese sind durch die Vorselaer-Wärmeschwankung voneinander getrennt. Ins ostwestfälische Untersuchungsgebiet gelangte nur der DrentheVorstoß, dessen äußerste Reichweite nach SERAPHIM (1972) und den Neukartierungen des Geologischen Landesamts Nordrhein-Westfalen in Abbildung 2 dargestellt wird.

$\mathrm{Zu}$ Beginn der Saale-Kaltzeit verstärkte sich als Auswirkung des Periglazialklimas die mechanische Verwitterung der mesozoischen Festgesteine, und ihr Schutt wurde in den während der Elster-Kaltzeit stark eingetieften Tälern in der Position von Mittelterrassen abgesetzt. Diese Kiessandkörper wurden später durch den Vorschüttsand des heranrückenden Gletschers überdeckt und anschließend von diesem überfahren, so daß die Terrassenkonturen verwischt wurden und das Gesteinsmaterial sich mit den glazigenen Sedimenten vermengte. In den Seitentälern der Weser blieb der frühdrenthezeitliche Mittelterrassenkörper lediglich in Sedimentfallen erhalten.

So wurde ein zeitweiliger Werrelauf von Bad Salzuflen über Exter, Babbenhausen und Rehme zur damaligen Weser in den Subrosionssenken von Steinbeck-Loose, Exter-Krutheide, Babbenhausen und Großer Weserbogen anhand seines Geröllanteils an Teutoburger-Wald-Gesteinen identifiziert (LohSE 1988, Deutloff 1995b:74).

In der Subrosionssenke von Babbenhausen (Blatt 3719 Minden) kam im Hangenden dieser WerreMittelterrasse sogar Niedermoortorf mit Holzresten zur Ablagerung, der jedoch nur eine nicht datierbare boreale Pollenflora enthielt. Als älteste glazigene Ablagerung des Drenthe-Stadiums blieben in dieser Senke feingeschichtete Beckensilte 
$\simeq$

Tाता

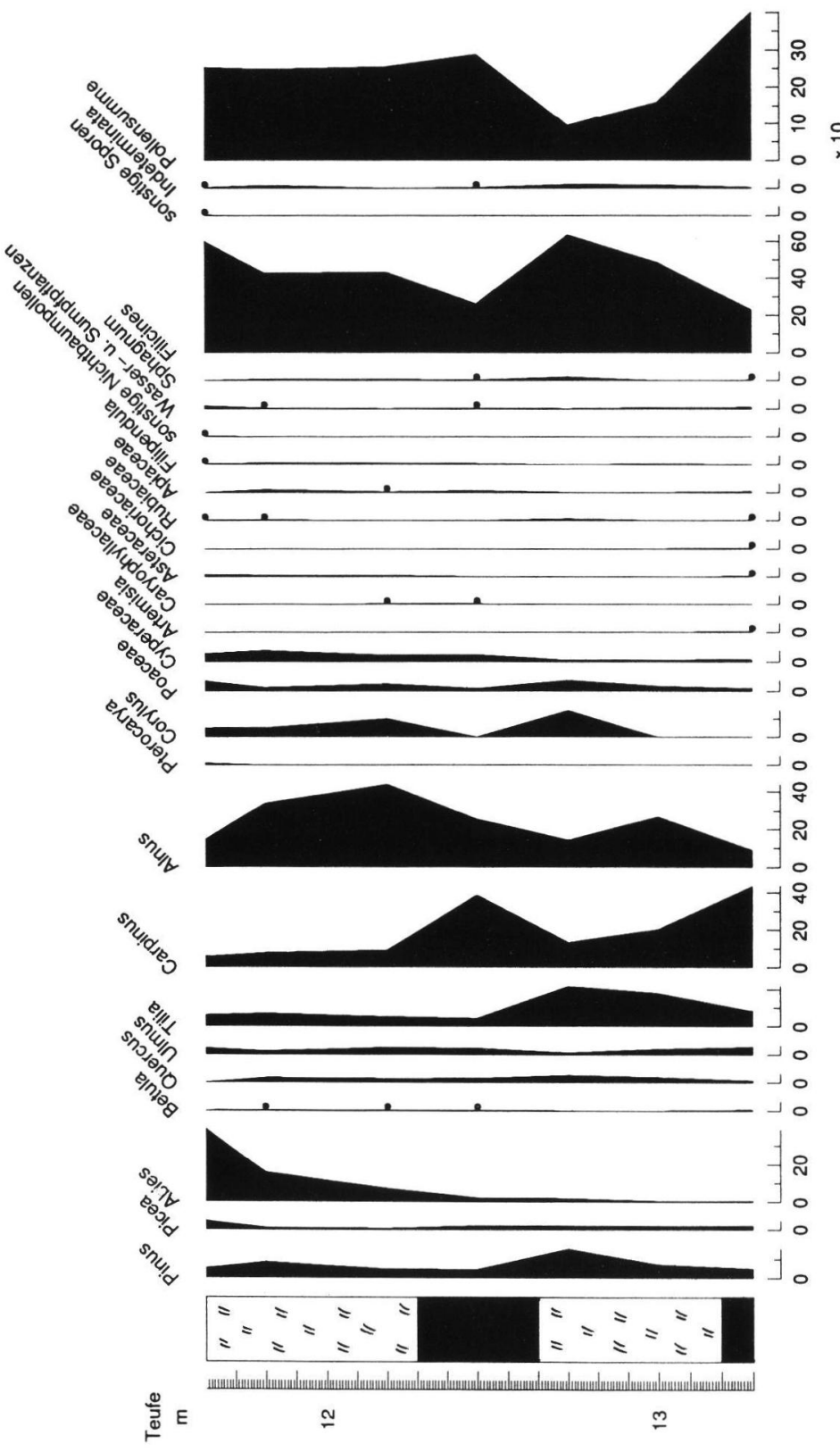

Abb. 8: Pollendiagramm des eemzeitlichen Abschnitts im Profil Ziegelei Bergmann. Schwarze Punkte markieren Pollenprozente unter $2 \%$.

Fig. 8: Pollen diagram of Eemian age in the section Brickyard Bergmann. Black dots indicate pollen percentages below $2 \%$.

des sogenannten Rintelner Eisstauseesystems erhalten (Kulle 1985, LOHSE 1988), über denen geringmächtiger Vorschüttsand und Geschiebemer- gel folgen. Im Blattgebiet 3818 Herford erschloß die kombinierte Schappen- und Kernbohrung Steinbeck 1976 (Abb. 3 Nr. 8) in der Subrosionssenke von SteinbeckLoose die mit $65 \mathrm{~m}$ bisher mächtigste und vollständigste in Ostwestfalen erbohrte drenthezeitliche Schichtenfolge, näher beschrieben bei Deutloff (1995b: 162). In dieser Senke folgte danach die Auslaugung des Zechsteinsalzes während der Saale-Kaltzeit, wie es beispielsweise DUPHORN (1986: 113) für den Salzstock Gorsleben in Niedersachsen beschrieben hat.

\subsection{Eem-Warmzeit}

Ablagerungen aus der Eem-Warmzeit innerhalb des drenthezeitlichen Vereisungsgebiets lassen sich nicht nur durch ihre charakteristische Pollenflora, sondern auch durch ihre Position im Hangenden der glazigenen Sedimente sowie eine markante nordische Komponente in ihrer Kiesfraktion identifizieren, jedoch sind Tagesaufschlüsse im Weserbergland sehr selten. Nur zwei derartige pollenanalytisch datierte Schichtenfolgen wurden bisher beschrieben, nämlich der Steiluferaufschluß an der Werre bei Nienhagen, Blatt 4018 Lage (SchütrumpF 1980: 70) sowie eine geringmächtige Abfolge von Torf, Mudde und humosem silt über drenthestadialen Sedimenten in einer Sandgrube östlich Buer, Blatt 3716 Melle (MEyer K.-D. \& MEYER, K.-J. 1992: 81). Kernbohrungen, die 1996 abgeteuft worden waren, erbrachten den Nachweis, daß der erstgenannte Aufschluß in einer Subrosionsenke (Subrosionssenke von Nienhagen, Abb. $2 \mathrm{Nr}$. 16) gelegen ist, deren mindestens $26 \mathrm{~m}$ mächtige Abschwemm-Massen auch holsteinzeitliche und unterpleistozäne Sedimente enthalten (MANTHEY 1997).

Günstigere Erhaltungsbedingungen boten wiederum die Sedimentfallen der Auslaugungssenken. Die wichtigste ist diejenige der Ziegelei 
Bergmann in Kalletal-Hohenhausen (Blatt 3819 Vlotho), die im Grenzbereich der drenthezeitlichen Vereisung liegt. In dieser Ziegeleitongrube wurde zur Zeit der Erstkartierung ein dunkelgrauer zäher, humoser Ton mit Torflagen abgebaut, den NaumanN (1922) als terrestrisches, Braunkohlen führendes Miozän ansprach. Durch neuere Untersuchungen wurde die Schichtenfolge als Pleistozän-Füllung einer Auslaugungssenke erkannt, und 1989 lieferten zwei Schappenbohrungen für Grundwassermeßstellen geeignetes Probenmaterial für die Pollenanalyse. Das knapp $7 \mathrm{~m}$ mächtige torfhaltige bis humose Profil (Abb. $3 \mathrm{Nr}$. 9) mit siltigen sowie sandig-kiesigen Partien zeigt eine Florenabfolge des ausgehenden Eems (Abb. 8). Der anfangs dominierende Eichenmischwald, in dem auch Carpinus stockte, wurde infolge einer Klimaabkühlung durch Nadelgehölze (Pinus, Abies) in zunehmendem Maße verdrängt. Typisch für das Eem sind auffallend hohe Tilia-Anteile im Eichenmischwald sowie hohe Abies-Quoten unter den Nadelgehölzen.

Weder die sandig-kiesigen Lagen innerhalb des eemzeitlichen Profils noch der geringmächtige Keuper-Kies der unterlagernden WesterkalleMittelterrasse enthalten allerdings nordische Gesteine.

Innerhalb des drenthezeitlichen Vereisungsgebiets liegt die Subrosionssenke Großer Weserbogen (Blatt 3719 Minden), die 1987 durch eine kombinierte Spül- und Rammkernbohrung erkundet wurde, nachdem bei der Naßauskiesung dort eine extrem hohe Mächtigkeit der WeserNiederterrasse angetroffen worden war. Die Bohrung Großer Weserbogen 1987 (Abb. 3 Nr. 10) erschloß im Liegenden der $28 \mathrm{~m}$ mächtigen Hochflut- und Niederterrassenablagerung $15,8 \mathrm{~m}$ überwiegend siltig-tonige, humose, jedoch kalkhaltige bis kalkreiche Abschwemm-Massen, die nur in den obersten $4 \mathrm{~m}$ eine ausreichend repräsentative Pollenflora lieferten: aus $28,50 \mathrm{~m}$ Teufe ein Pollenspektrum der Kiefern-Zeit, aus 31,9 m Teufe ein solches der Kiefern-Fichten-Tannen-Zeit innerhalb des Eems (Bearb.: H.-W. ReHAGEN Geol. L.-Amt Nordrh.-Westf.). Die Pollengemeinschaften können mit den Zonen VII und VI des Profils von Rederstall in Dithmarschen, Schleswig-Holstein (Menke \& Tynni 1984) parallelisiert werden.

Die Geröllanalyse der eingeschalteten Kiese ergab eine starke Vormacht von Weserberglandgesteinen (74-90\%) vor nordischen (bis 18\%), während Material des Wesertals fehlte (LoHSE 1988). Dieses Ergebnis stützt die pollenstratigraphische Datierung.

\subsection{Weichsel-Kaltzeit}

Während des Weichsels gehörte das Untersuchungsgebiet zum Periglazialbereich weit südlich des Eisrandes. In den Tälern sedimentierten Kiese und Sande der Niederterrassenkörper. Weitflächig wurde siltig-feinsandiger Löß aufgeweht, und in den Hanglagen bildeten sich Fließerden aus pleistozänen Lockergesteinen und Festgesteinsschutt. Nach den bisherigen Bohrbefunden im Untersuchungsgebiet entstanden keine Subrosionssenken neu, doch senkten sich einige bereits vorhandene erneut ein und wurden mit Sediment gefüllt. Dabei entstanden vor allem besonders mächtige Schotterkörper der Niederterrassen im Weser- und Werretal.

In der Subrosionssenke Großer Weserbogen wurde 23,0 m mächtiger Niederterrassen-Kies erbohrt: Zuunterst lagert $8,0 \mathrm{~m}$ sandiger Fein- bis Grobkies mit Lagen von tonigem Silt, darüber folgen 8,0 m feinkiesiger Mittelkies und schließlich $7,0 \mathrm{~m}$ sandiger Fein- und Mittelkies. Nur die beiden oberen Abschnitte weisen einen deutlichen Kalkgehalt auf. Im Geröllspektrum herrschen Buntsandstein und paläozoisches Material des Wesertals gegenüber mesozoischen Gesteinen des Weserberglands vor, der Anteil nordischer Geschiebe schwankt zwischen 3 und $9 \%$ (LoHSE 1988).

Die Niederterrassen-Mächtigkeit im Großen Weserbogen ist zwar die größte bisher im nordrheinwestfälischen Abschnitt des Wesertals zwischen Rinteln und Porta Westfalica nachgewiesene, doch wird sie von der Füllung einer ausgedehnten Subrosionssenke im Raum Hameln in Niedersachsen weit übertroffen. Dort und weseraufwärts bis Bad Karlshafen sind entsprechende Untersuchungen derzeit im Gang (J. LePper und W. THIEM, mdl. Mitt.)

In der Subrosionssenke Schlachthof Bad Oeynhausen werden die bavelzeitlichen AbschwemmMassen von 14,8 m Fein- bis Grobsand mit eingeschalteten dünnen Kieslagen der Werre-Niederterrasse überlagert, die dort ihre größte bisher im Stadtgebiet bekanntgewordene Mächtigkeit besitzt. Im Geröllspektrum herrschen heimische Gesteine aus Weserbergland und Teutoburger Wald gegenüber nordischen wiederum stark vor, doch erreichen letztere jeweils Anteile zwischen 13 und $20 \%$ (LOHSE 1988).

Am Nordrand des Werretales wurden in der wohl eemzeitlich eingesunkenen Subrosionssenke Blutwiese in Löhne-Gohfeld (Abb 2 Nr. 14) zwischen zwei Niederterrassen-Kieskörpern und im Liegenden davon pollenführende Silte und Tone 
erbohrt (Henke 1990), die nach H.-W. ReHAgen (unveröff. Bericht Geol. L.-Amt Nordrh.-Westf.) wahrscheinlich dem Odderade- bzw. dem Moershoofd-Interstadial des Weichsel-Frühglazials zuzuordnen sind.

Weichselzeitlicher Löß fand sich in der für das Blattgebiet 3818 Herford maximalen Mächtigkeit zwischen 7,6 und 8,6 $\mathrm{m}$ in den Subrosionssenken von Hollwiesen-Horst und Steinbeck-Loose (Deutloff 1995b: 88).

\section{Ergebnis}

Mit den beschriebenen Schichtenfolgen aus 10 Subrosionssenken werden erstmals die aus diesen Sedimentfallen gewonnenen neuen Erkenntnisse über die geologische Geschichte Ostwestfalens während des Pleistozäns im Zusammenhang vorgestellt. Diesen Ereignissen ging in den ältesten Subrosionssenken eine langfristige tertiärzeitliche Entwicklung voraus, die beispielsweise in der Senke Kurpark Vlotho, Blatt 3819 Vlotho (Abb. 2 Nr. 11), im Unteroligozän begann (DeuTLOFF 1995a).Andererseits dauerten die Senkungsbewegungen in einigen Strukturen auch während des Holozäns an oder setzten nach Ende des Weichsels von neuem ein, so in den Subrosionssenken von Exter-Krutheide und Steinbeck-Loose, Blatt 3818 Herford (Deutloff 1995: 91 u. Tab. 11) beziehungsweise Hücker Moor, Blatt 3817 Bünde (Freund 1994).

Aus den gewonnenen Erkenntnissen läßt sich folgern, daß

1. die Subrosion in Ostwestfalen spätestens zu Beginn des Oligozäns einsetzte und das gesamte jüngere Tertiär und das Quartär hindurch andauerte, allerdings mit unterschiedlicher Intensität.

2. in den Subrosionssenken die AbschwemmMassen fast ausschließlich im limnisch-fluviatilen Milieu sedimentiert wurden. Nur selten kam es zum Aufwuchs von Niedermooren während Ruhepausen der Absenkung. Daher schwankt der Anteil organischen Materials in den Sedimenten innerhalb weiter Grenzen, wodurch die pollenanalytische Altersbestimmung allzuoft stark eingeschränkt wird.

3. das pleistozäne Subrosionsgeschehen seinen Höhepunkt während des Cromer-Komplexes erreichte.

4. entgegen einer früher verbreiteten Meinung die Subrosionsprozesse auch während der pleistozänen Kaltzeiten andauerten, wie das Beispiel der Bohrung Steinbeck 1976 für die Saale-Zeit be- weist. Um so auffälliger ist die Abwesenheit glazigener Ablagerungen aus der vorangegangenen Elster-Kaltzeit in sämtlichen ostwestfälischen Subrosionssenken außer derjenigen von Mühlenhof Möllbergen nahe der Porta Westfalica.

5. der Nachweis oberpleistozäner Sedimente in Subrosionssenken des Weser- und Kalletals eine Nordwanderung der Subrosionsvorgänge anzudeuten scheint, doch fehlen für eine zuverlässige Aussage noch entsprechende Untersuchungen im südlichen Weser- und Wiehengebirgsvorland.

\section{Danksagung}

Da die meisten der beschriebenen Forschungsbohrungen durch das Geologische Landesamt Nordrhein-Westfalen ausgeführt wurden, waren an deren Bearbeitung zahlreiche Kolleginnen und Kollegen beteiligt, denen hiermit gedankt sei. Besonders erwähnt sei Herr Dipl.-Ing. J. RoTHER, der bei den Bohrungsbearbeitungen tatkräftig und zuverlässig mitwirkte.

Prof. Dr. GRüGER/Göttingen sei für palynostratigraphische Diskussionsbeiträge herzlich gedankt.

\section{Schriftenverzeichnis}

Becker, L. (1975): Das Vahlhäuser Senkungsfeld, eine mittelpleistozäne Subrosionssenke im Meinberger Graben (östlich Detmold). - N. Jb. Geol. Paläont., Abh., 150: 373 - 388, 3 Abb., 3 Tab.; Stuttgart.

Benda, L.; Gaertner, H. R. von; Herrmann, R.; Lüttig, G.; Streif, H.; Wunderlich, H. G. (1968): Känozoische Sedimente in tektonischen Fallen und Subrosionssenken in Süd-Niedersachsen. - Z. dt. Geol. Ges., 117: 713 - 726, 1 Abb., 1 Tab.; Hannover.

BenNet, K. D. (1994): PSIMPOLL version 2.23: a C program for analysing pollen data and plotting pollen diagrams. - Newsl. INQUA Working Group Data Handling Methods, 11: 1-3; Oxford.

Caspers, G.; Jordan, H.; Merkt, J.; Meyer, K.-D.; Müller, H.; Streif, H. (1995): Niedersachsen. - In: Benda, L. [Hrsg.]: Das Quartär Deutschlands: 23 - 58, 9 Abb., 1 Tab.; Berlin, Stuttgart (Borntraeger). - [Internat. Kongr. Quart.-Vereinig. <4., 1995, Berlin>].

Deutloff, O. (1995a): Forschungsbohrung Kurpark Vlotho 1993. - In: Geowissenschaftliche Gemeinschaftsaufgaben. Tätigkeitsbericht 1993/94: 89 - 91, 1 Abb., 1 Tab.; Hannover (Niedersächsisches Landesamt Bodenforschung).

- (1995b), mit Beitr. von Dubber, H.-J.; JäGer, B.; MIChel, G.; Vieth-Redemann, A.: Erläuterungen zu Blatt 3818 Herford. - Geol. Kt. Nordrh.-Westf. 1:25 000, Erl., 3818, 2. Aufl: 182 S., 13 Abb., 17 Tab., 2 Taf.; Krefeld.

- Kühn-Velten, H.; Michel, G.; Skupin, K. (1982): Erläuterungen zu Blatt C 3918 Minden. - Geol. Kt. Nordrh.-Westf. 1:100 000, Erl., C 3918: 80 S., 17 Abb., 2 Tab.; Krefeld. 
- Kühn-Velten, H.; Michel, G. (1986): Erläuterungen zu Blatt C 3914 Bielefeld. - Geol. Kt. Nordrh.-Westf. 1:100 000, Erl., C 3914: 104 S., 19 Abb., 2 Tab.; Krefeld.

DuphoRn, K. (1986): Das subrosive Sicherheitsrisiko bei der geplanten Endlagerung von radioaktiven Abfällen im Salzstock Gorleben aus quartärgeologischer Sicht. - Z. dt. geol. Ges., 137: 105-120, 5 Abb.; Hannover.

EIssmanN, L. (1994): Grundzüge der Quartärgeologie Mitteldeutschlands (Sachsen, Sachsen-Anhalt, Südbrandenburg, Thüringen). - Altenburger naturwiss. Forsch., 7: 55 - 135, 35 Abb., 5 Tab., 12 Taf.; Altenburg.

FAEGri, K.; Iversen, J. (1993): Bestimmungsschlüssel für die nordwesteuropäische Pollenflora. - 85 S., 453 Abb.; Jena.

Farrenschon, J. (1986), mit Beitr. von Dahm-Ahrens, H.; Michel, G., Vogler, H.: Erläuterungen zu Blatt 4019 Detmold.- Geol. Kt. Nordrh.-Westf. 1: 25 000, Erl., 4019, 2. Aufl.: 172 S., 13 Abb., 15 Tab., 3 Taf.; Krefeld.

- (1995a): Loam pit „Bergmann“ in Blomberg-Hohedömsen. - In: Schirmer, W. [Hrsg.]: Quaternary field trips in Central Europe. 1; Regional field trips 10: Central Upland Margin Traverse, Stop 26: 586 - 587 , 1 Abb.; München (Pfeil-Verl.).

- (1995b), mit Beitr. von Hoffmann, M.; Michel, G.; WARSTAT, M.: Erläuterungen zu Blatt 4020 Blomberg.- Geol. Kt. Nordrh.-Westf. 1: 25 000, Erl., 4020 , 2. Aufl.: 155 S., 9 Abb., 13 Tab., 1 Taf.; Krefeld.

- (1998), mit Beitr. von Betzer, H.-J.; JÄGer, B.; Michel, G.: Erläuterungen zu Blatt 3919 Lemgo.- Geol. Kt. Nordrh.-Westf. 1 : 25 000, Erl., 3919, 2. Aufl.: 150 S., 8 Abb., 10 Tab., 1 Taf.; Krefeld.

- (1998): Ursachen und Folgen großräumiger Hohlraumbildungen durch Salinarkarst und Salzabwanderung im tiefen Untergrund des Lippischen Berglandes. - Lippische Mitt. Gesch. u. Landeskde., 67: 283-308, 7 Abb., 3 Tab.; Detmold.

Freund, H. (1994): Pollenanalytische Untersuchungen zur Vegetations- und Siedlungsentwicklung im westlichen Weserbergland. - Abh. Westf. Mus. Naturkde., 56: 3-103, 35 Abb., 6 Tab.; Münster.

Fromm, K. (1994): Paläomagnetische Bestimmungen an Quartärsedimenten in Nordwest-Deutschland. Geol. Jb., A, 134: 229 - 257, 13 Abb., 1 Tab.; Hannover.

GEOLOGISCHES LANDESAMT NORDRHEIN-WESTFALEN [Hrsg.] (1986): Tätigkeitsbericht 1984 bis 1985.86 S., 71 Abb., 12 Tab.; Krefeld.

Hahne, J.; Mengeling, H.; Merkt, J.; Gramann, F. (1994): Die Hunteburg-Warmzeit („Cromer-Komplex") und Ablagerungen der Elster-, Saale- und Weichsel-Kaltzeit in der Forschungsbohrung „Hunteburg GE 58“ bei Osnabrück. - Geol. Jb., A, 134: 117 - 165, 21 Abb., 4 Tab.; Hannover.

Henke, J.-H. (1990): Die Niederterrassenablagerungen der Werre in der Flur „Blutwiese“, Löhne-Gohfeld. Ber. naturwiss. Ver. Bielefeld, 31: 71 - 83, 4 Abb., 1 Tab.; Bielefeld.
Herrmann, R. (1968): Auslaugung durch aufsteigende Mineralwässer als Ursache von Erdfällen bei Bad Pyrmont. - Geol. Jb., 87: 265 - 284, 8 Abb., 1 Taf.; Hannover.

— (1972): Über Erdfälle äußerst tiefen Ursprungs (Die „Wolkenbrüche“ bei Trendelburg und die „Meere“ bei Bad Pyrmont). - Notizbl. hess. L.-Amt Bodenforsch., 100: 177 - 193, 5 Abb., 1 Taf.; Wiesbaden.

Homann, M.; Lepper, J. (1994): Das Cromer-Profil von Sohlingen (Süd-Niedersachsen). - Geol. Jb., A, 134: 211 - 228, 5 Abb., 1 Tab.; Hannover.

Jordan, H.; Schwartau, W. (1993): Das Lößprofil von Ahlshausen und weitere tiefe Quartäraufschlüsse entlang der Bundesbahn-Neubaustrecke bei Northeim, Südniedersachsen. - Eiszeitalter u. Gegenwart, 43: 110 - 122, 9 Abb., 3 Tab.; Hannover.

Kaltwang, J. (1992): Die pleistozäne Vereisungsgrenze im südlichen Niedersachsen und im östlichen Westfalen. - Mitt. Geol. Inst. Univ. Hannover, 33: 161 S., 7 Abb., 38 Tab., 49 Kt.; Hannover.

Klostermann, J. (1995): Nordrhein-Westfalen. - In: Benda L. [Hrsg.]: Das Quartär Deutschlands: 59 - 94, 6 Abb., 8 Tab.; Berlin, Stuttgart (Borntraeger).- [Internat. Kongr. Quart.- Vereinig. <4., 1995, Berlin>].

Knauff, W. (1978):, mit Beitr. von Deutloff, O.; JäGer, B.; Michel, G.; WILl, K.-H.: Erläuterungen zu Blatt 3918 Bad Salzuflen.- Geol. Kt. Nordrh.-Westf. 1:25000, Erl., 3918, 2. Aufl.: 143 S., 17 Abb., 18 Tab., 5 Taf.; Krefeld.

Knoth, W. (1995): Sachsen-Anhalt. - In: BEnDA, L. [Hrsg.]: Das Quartär Deutschlands: 148 - 170, 5 Abb., 4 Tab.; Berlin, Stuttgart (Borntraeger).- [Internat. Kongr. Quart.- Vereinig. <4., 1995, Berlin>].

Kulle, S. (1985): Drenthestadiale (pleistozäne) Staubeckensedimente und ihr Lagerungsverband in zwei Aufschlüssen im Wesertal zwischen Rinteln und Hameln. - Dipl.-Arb. Univ. Hannover: 82 S., 18 Abb., 8 Tab., 4 Taf., 3 Anl.; Hannover. - [Unveröff.]

LoHsE, D. (1988): Quartärgeologische Auswertung von Flachbohrungen im Raum Bad Oeynhausen - Vlotho, mit einer Kartierung am Westhang des Buhn. Dipl.-Arb. u. -Kart. Univ. Hannover: 58 S., 11 Abb., 3 Tab., 30 Prof. im Anh., 2 Prof. u. 2 Kt. in Anl.; Hannover. - [Unveröff.]

LÜTTIG, G. (1969): Abnorme Quartärprofile im nordwestdeutschen Bergland. - Geol. Jb., 88: 13 - 34, 5 Abb., 2 Tab.; Hannover.

MANIA, D. (1967): Das Jungquartär aus dem ehemaligen Ascherslebener See im Nordharzvorland. - Petermanns geogr. Mitt., 111: 257 - 273, 11 Abb., 4 Tab., 5 Taf.; Gotha, Leipzig.

MANTHEY, S. (1997): Quartärgeologische Kartierung westlich von Detmold zwischen Werre und Teutoburger Wald. - Dipl.-Kart. Univ. Münster: VII+62 S., 20 Abb., 4 Tab., 4 Anl., Anh.; Münster. - [Unveröff.] Menke, B. (1975): Vegetationsgeschichte und Florenstratigraphie Nordwestdeutschlands im Pliozän und Frühquartär. Mit einem Beitrag zur Biostratigraphie des Weichselfrühglazials. - Geol. Jb., A, 26: 3 - 151, 9 Abb., 3 Tab., 8 Taf.; Hannover. 
- Trnni, R. (1984): Das Eeminterglazial und das Weichselfrühglazial von Rederstall/Dithmarschen und ihre Bedeutung für die mitteleuropäische Jungpleistozän-Gliederung. - Geol. Jb., A 76: 3-120, 18 Abb., 7 Tab., 9 Taf.; Hannover.

Mestwerdt, A. (1951): Zur Entstehung des Hücker Moores. - Festschr. z. 800-Jahrfeier der Gemeinden Hücker - Aschen, Kreis Herford, 1951: 7 S.; Herford.

Meyer, K.-D.; Meyer, K.-J. (1992): Das Eem-Interglazial von Buer bei Melle/Osnabrüick. - Osnabrücker naturwiss. Mitt., 18: 81 - 90, 5 Abb., 1 Tab.; Osnabrück.

Moore, P. D.; WebB, J. A. Collinson, M. E. (1991): Pollen Analysis. - 216 S.; Oxford.

MülLER, H. (1974): Pollenanalytische Untersuchungen und Jahresschichtenzählungen an der holsteinzeitlichen Kieselgur von Munster-Breloh. - Geol. Jb., A 21: 107 - 140, 10 Abb., 2 Tab.; Hannover.

NaumanN, E. (1922): Erläuterungen zu Blatt Vlotho. Geol. Kt. Preußen u. benachb. B.-Staaten 1 : 25 000, Erl., 3819: 54 S., 1 Abb.; Berlin.

NIEDERSÄCHSISCHES LANDESAMT FÜR BODENFORSCHUNG (1995): Quartärgeologische Übersichtskarte von Niedersachsen und Bremen 1 : 500 000.Bearb. Hinze C.; Höfle, H.-C.; Jordan, H.; MengeLING, H.; MEYer, K.-D. (Redaktion), RoHde, P.; STREIF, H.; Hannover.

Rehagen, H.-W. (1980): Pollenanalytische Untersuchungsergebnisse aus dem Pleistozän in Ostwestfalen- Lippe. - Westf. geogr. Stud., 36: 57 - 64, 2 Tab.; Münster/Westf. [Tagung Arb.-Gem. Nordwestdeutscher Geologen <46., 5.-8.Juni 1979, Münster>].

RÖHM, H. (1985): Bau und Zusammensetzung Saale-Eiszeitlicher Sedimentkörper im Raum Hausberge Veltheim/Weser. - Dipl.-Arb. u. -Kart. Univ. Hannover: 107 S., 53 Abb., 7 Anl.; Hannover. - [Unveröff.]

Rohde, P. (1994): Weser und Leine am Berglandrand zur Ober- und Mittelterrassen-Zeit. - Eiszeitalter u. Gegenwart, 44: 106-113, 2 Abb.; Hannover.
SCHNEIDER, H. (1975): Subrosionssenken im nordwestfälischen Bergland. - Münstersche Forsch. Geol. Paläont., 35: 71 - 88, 5 Taf.; Münster/Westf.

SchütrumpF, R. (1980): Das Interglazial im Werre-Profil bei Nienhagen (Detmold). - Westf. geogr. Stud., 36: 65 - 70, 5 Abb.; Münster/Westf. [Tagung Arb.-Gem. Nordwestdeutscher Geologen <46., 5.-8.Juni 1979, Münster>].

Seraphim, E. Th. (1972): Wege und Halte des saalezeitlichen Inlandeises zwischen Osning und Weser. Geol. Jb., A, 3: 85 S., 14 Abb., 6 Tab.; Hannover.

Skupin, K.; SpeEtzen, E.; Zandstra, J. G. (1993): Die Eiszeit in Nordwestdeutschland: Zur Vereisungsgeschichte der Westfälischen Bucht und anschließender Gebiete. - 143 S., 49 Abb., 24 Tab., 2 Taf., 2 Kt.; Krefeld (Geol. L.-Amt Nordrh.-Westf.).

Stephan, H.-J.; Menke, B. (1994): Das Pleistozän in Schleswig-Holstein. - GLA SH, 3: 19-62, 8 Abb., 33 Tab.; Kiel (Geol. L.-Amt).

Weber, H. (1952): Pliozän und Auslaugung im Gebiet der oberen Werra. - Geologica, 8: 136 S., 17 Abb., 4 Fototaf.; Berlin.

Wellmann, P. (1990): Aufbau und Genese des Saale-eiszeitlichen Sedimentkörpers von KrankenhagenMöllenbeck. - Dipl.-Arb. u. -Kart. Univ. Hannover: 84 S., 45 Abb., 1 Tab., 13 Anl.; Hannover. - [Unveröff.]

ZaGWIJN, W. H. (1957): Vegetation, climate and timecorrelations in the early Pleistocene of Europe. Geol. en Mijnb., N. S., 19: 233 - 244, 4 Abb., 2 Tab.; 's-Gravenhage.

- (1985): An outline of the Quaternary stratigraphy of the Netherlands. - Geol. en Mijnb., 64: 17 - 24, 5 Abb.; Dordrecht.

Zubrägel, A. (1991): Paläomagnetische Untersuchungen an Sedimenten einer Subrosionssenke im Salzetal. - Dipl.-Arb. Univ. Münster: 77 S., 41 Abb., 4 S. Anl.; Münster. - [Unveröff.]

Manuskript eingegangen am 19. April 1999 